COMPTES RENDUS

\title{
COURANTS PHILOSOPHIQUES
}

Raymond Polin, La Création des cultures. D'une philosophie de l'histoire à une philosophie des cultures. Paris, Presses universitaires de France, 1993. $15 \times 21,6,282$ p. («Questions »).

Ce volume débute par un "adieu à la philosophie de l'histoire». Mais, en récusant l'épure du type idéal des philosophies de l'histoire qu'ont forgée, de Rousseau à Kant et à Hegel, les philosophes modernes, R. Polin ouvre le livre prestigieux dans lequel se dessinent en traits vivants des histoires placées sous le double signe de la pluralité et de la diversité. Ce basculement de la pensée s'explique aisément. En effet, depuis le XvIII ${ }^{e}$ siècle, la philosophie de l'histoire trouve son principe fondamental dans la définition abstraite de la liberté comme capacité de perfectibilité. La liberté ainsi conçue appelant une finalité et impliquant une téléologie, la philosophie de l'histoire est tendue, à raison de sa logique interne, vers « la fin de l'histoire ». Or, même si l'on admet qu'un tel schéma procède d'une reconstitution conceptuelle, il faut reconnaître, devant la force des choses, « l'incomparable pouvoir de faire illusion » (p. 12) qui vicie une telle démarche. Rousseau, déjà, avait renoncé à parler d'une « société générale du genre humain » caractérisée par l'unité, la solidarité, des caractères et un droit universels. Aujourd'hui, de multiples recherches - philologiques, ethnologiques, sociologiques, historiques, économiques... - nous enseignent qu'il existe une "irréductible pluralité des cultures " (p. 13) : sociétés, peuples, nations, groupes et individus se signalent par leur diversité et leur différence. Sans que l'unité de l'espèce humaine soit jamais mise en question, il y a, plutôt qu'une Histoire (comme si l'espèce humaine se développait selon une évolution unilinéaire), "une multitude d'histoires, qui parfois se rencontrent et interfèrent, qui naissent, chacune sur une aire donnée, de la composition et de la convergence pratiques d'un ensemble d'efforts humains » (p. 24). L'invention des philosophies de l'histoire n'est donc rien d'autre, devant l'expérience vécue, qu'un « arbitraire passage à l'absolu ». Elle correspond au « mythe d'une histoire totale imaginaire appliqué au mythe d'une société humaine totale imaginaire ». Aussi est-il grand temps de comprendre que chacun des ensembles historiques dont il faut reconnaître l'incompressible réalité objective est le creuset de cultures différenciées qui s'offrent comme un ensemble de valeurs créées au fil du temps, d'actes et d'œuvres accomplis dans la longue durée.

Revue de synthèse : $4^{\text {e }} \mathrm{S} . \mathrm{N}^{\mathrm{os}}$ 3-4, juil.-déc. 1996, p. 503-575. 
Prolongeant la méditation qu'il avait conduite en 1944 dans La Création des valeurs, R. Polin propose aujourd'hui une réflexion sur ces «cultures» en quoi s'exprime, dit-il, une liberté qui, loin d'être un commencement, n'est et ne vaut que par ce qu'elle fait.

La liberté étant définie comme un pouvoir de création, $R$. Polin en considère l'action et le produit comme une réalité originale que, précisément, il désigne sous le nom de culture. À la différence de la civilisation, la culture désigne « l'aspect spirituel de la vie d'une société à chaque moment de son histoire ». Constituée par « une myriade d'esprits en acte », elle se fonde à partir de l'affirmation de valeurs, de fins et de normes; elle s'incarne dans les mceurs, se manifeste par des coutumes, des traditions, des institutions mais aussi par des convictions, des religions, des passions... d'où naissent des mythes, des dogmes et des règles. De cette « accumulation d'œuvres », se dégage « un style propre » dont l'originalité ne demande rien à l'illusoire Weltgeist. Dès lors, dans un monde où « il n'y a ni vérité des valeurs ni donc universalité des valeurs » (p. 66) - «le concept de valeur est incompatible avec celui d'universalité » (p. 84) —, la communicabilité entre les cultures dont chacune est «une création continuée foisonnante » (p. 49) fait surgir des problèmes complexes. Sous le signe du relativisme des valeurs et du pluralisme des cultures qui en découle, R. Polin traite du «principe » qui suscite ces problèmes et des efforts pour les résoudre.

La problématique de la communication des cultures - aujourd'hui plus redoutable que jamais dans un contexte mental d'où trop souvent sont bannies les « idées claires et distinctes »- suppose, au principe de la recherche, l'analyse phénoménologique de la culture. Il importe en effet de n'en point confondre le concept avec celui de civilisation. Dans le très beau chapitre intitulé «Culture et Civilisation », l'auteur, ayant retracé l'émergence de la civilisation occidentale dominée par la rationalité technicienne, souligne fortement que « la civilisation qui se séparerait de la culture dont elle a émergé pour la servir, quand elle atteint un certain stade de réussite, semble ne plus se propulser que par sa seule inertie, et prend le risque de devenir alors un monstre d'absurdité qui n'est plus maîtrisable " (p. 119). Mesurons par conséquent ce qu'a de désastreux la notion de «culture de la communication » dont la mode intellectuelle s'est aujourd'hui emparée : elle n'est que «pseudoculture », voire « contre-culture ». La véritable culture n'a pas le caractère réducteur qu'impose la technique mise en cuvre dans l'éducation électronique des masses où triomphe l'endoctrinement idéologique, ou dans la communication audio-visuelle qui, dans l'uniformité, rabaisse l'homme à l'état de spectateur. Elle a besoin, au contraire, du temps de la réflexion; elle vit de la mémoire du passé et de l'imagination de l'avenir; elle a besoin de jugements, de croyances et de normes; elle est « une culture de la liberté par la liberté " (p. 132). Tandis que la civilisation est affaire d'intelligence et de raison, la culture est affaire d'esprit et de liberté. C'est pourquoi, s'il est loisible de concevoir une civilisation universelle des sciences et des techniques, celle-ci, qui n'est pas une culture, ne peut ni inspirer ni fonder un ordre politique universel : « Il ne peut y avoir d'ordre politique universel parce qu'il n'y a pas et qu'il ne peut y avoir de culture universelle de l'humanité » (p. 138).

Il faut compter aussi avec le « devenir des cultures » auquel R. Polin consacre le chapitre iv de son livre. L'homme, être de mémoire et d'avenir, être social et historique, se trouve toujours en acte «entre deux ceuvres» et « en transcendance à 
l'égard de lui-même ». Tout ce qui est d'ordre culturel traduit par conséquent, dans la coexistence d'une communauté, le pouvoir de dépassement de la liberté, ce par quoi l'homme devient véritablement homme. Aussi la culture peut-elle être considérée comme une œuvre d'art avec ce qu'elle a d'imprévisible, de vivant, d'aléatoire et de fragile. Dans ces conditions, on ne saurait appliquer l'idée de progrès au devenir d'une culture comme on l'applique au devenir des sciences et des techniques. Le progrès est un développement vers une fin. Or, penser qu'une culture, œuvre de la liberté créatrice, tend vers une fin est « un contresens aveugle» (p. 181). Une culture n'a pas de finalités ultimes; elle se développe en fonction de valeurs prédominantes eu égard auxquelles le rôle des élites - acteurs éminents de la liberté créatrice - est décisif. De la sorte, les caractères d'une culture lui sont spécifiques. Il s'ensuit que chaque culture étant « une ouvre tout entière tournée vers l'intérieur ", somme toute " solipsiste ", elle est, dans sa différence intime et essentielle, incommunicable. Il existe même une incomparabilité entre les cultures. Et, s'il est vrai que, sur ce fond d'incompatibilité, s'impose un devoir de respect envers l'égale dignité des diverses cultures, il est non moins vrai qu'il existe entre elles des « inégalités considérables » et une " hiérarchie ». On peut assurément reprocher à notre culture occidentale de s'être amplement illusionnée sur l'universalité de ses valeurs ou sur la perfection de ses institutions politiques. Ce sont là, à coup sûr, des erreurs et une démesure qu'il faut avoir le courage de condamner. Il reste cependant que " nulle autre culture n'a été capable de s'élever à un tel niveau d'excellence humaine dans tant de domaines, de former tant d'élites si éminentes et si diverses, de vivre enfin une si grande histoire» (p. 201).

Pourtant, de nos jours, se posent, sur ce fond d'incompatibilité souvent « fracassante », de redoutables problèmes que $R$. Polin envisage avec un regard lucide et pénétrant : ceux, par exemple, de l'intégration d'une culture à une autre ou de la cohabitation entre cultures au sein d'une même communauté. Surgit aussi la grave question de la possibilité de sociétés politiques multiculturelles. Avec une sagesse exemplaire, R. Polin, estimant qu'une espérance œcuménique ne serait pas utopique, élabore un «essai de déontologie » (p. 213) dont les règles raisonnables répondent à une idée directrice forte : elles ne sauraient relever d'un pouvoir politique extérieur car les affaires culturelles sont les affaires exclusives de la liberté. Aussi comprend-on le prix qui s'attache à la sauvegarde des cultures et, tout particulièrement, de notre culture d'Occident que mine la crise contemporaine.

Après avoir rappelé l'héritage par lequel notre culture a reçu des Grecs et des Romains, puis des juifs et des chrétiens, les valeurs fondamentales qui font la grandeur de l'humanisme, R. Polin s'interroge, avec sobriété mais clairvoyance, sur les turbulences, les troubles et les désordres qui mettent en péril les plus hautes valeurs de la culture occidentale. La liberté est menacée par un vent de "révolte pour la révolte », par le surréalisme ou l'anarchisme, par la confusion de la révolution avec la libération ou l'innovation, par l'idéologie de la bonté naturelle et le laxisme qui en dérive, par la réclamation de la justice sociale dispensée par l'État-providence... L'individualité, dans le torrent des libertés excessives et abusives que roule un amour de soi exclusif, est, elle aussi, entrée en crise comme l'avait pressenti Tocqueville. L'égalité, dont le concept implique la reconnaissance de la dignité et des mérites, s'est muée en une idéologie égalitaire qui ne trouve son effectuation, au mépris des valeurs et des élites, que dans un nivellement par le bas. Le régime de 
masse, qui se prétend l'apogée de la démocratie, sous-tendu par l'idéologie de l'humanité dont une certaine mode intellectuelle fait le vecteur d'une mondialisation politique et juridique, est également l'un des facteurs les plus néfastes pour la valeur de notre culture... Comment conjurer tant de menaces, sinon en faisant l'effort de comprendre d'abord que les institutions ne valent que ce que valent leurs membres, ensuite, que les requêtes métaphysiques sont en l'homme plus profondes que les conquêtes scientifiques? Tandis que le chemin du matérialisme conduit au triomphe de la civilisation, le chemin de la liberté est celui que « nous ouvre l'homme dont la nature consiste à faire librement sa nature » (p. 273). La tâche est incertaine et fragile; mais elle correspond à un devoir dont il importe d'affronter la difficulté.

Avec la générosité qui traverse toute son œuvre, R. Polin conclut que, à l'épicentre de la crise que nous vivons parfois jusqu'au drame, nous pouvons seulement «prendre [...] le risque d'un pari et exprimer notre foi, notre espérance dans les valeurs de l'esprit ».

En refermant ce livre, dont la modestie s'éclaire d'un rais puissant de lumière, nous pensons qu'une très belle leçon d'humilité et d'espérance est donnée à ceux qui, en cette fin de siècle, cèdent plus que jamais aux vertiges délétères des modes idéologiques. La création des cultures, comme la création des valeurs, porte témoignage de la capacité des hommes à la liberté. Seulement, le pluralisme des histoires et des cultures n'échappera, demain, à « la guerre des cultures» inscrite dans leur logique immanente que si les hommes assument le pari en faveur des valeurs de l'esprit. Dès lors, la grande question - qui n'est pas étrangère au souffle nietzschéen qui passe parfois sur l'œuvre philosophique de R. Polin - est de savoir si les hommes d'une trempe assez solide sont aujourd'hui et seront demain assez nombreux pour tenir ce pari, héroïque et pourtant nécessaire.

Simone GoYARD-FABRE

Charles Ramond, Qualité et Quantité dans la philosophie de Spinoza. Paris, Presses universitaires de France, 1995. 13,6 × 21,5, 332 p., bibliogr., index ( Philosophie d'aujourd'hui »).

L'ouvrage de Charles Ramond s'attache à montrer, d'une part, comment Spinoza s'est efforcé de penser la nature naturante du point de vue de la qualité et la nature naturée du point de vue de la quantité; et, d'autre part, pourquoi celui-ci n'a pas réussi totalement dans cette entreprise. Le plan adopté par $C$. Ramond, qui veut souligner le " caractère fondamentalement binaire, non dialectique, de la pensée de Spinoza », est le suivant : la qualité et la quantité sont étudiées d'abord en Dieu (première partie), où il est question du problème des «degrés de réalité et de perfection » (première section) et du problème de la nature de l'infini (seconde section); puis elles sont étudiées dans le domaine des modes finis (seconde partie), où les essences des choses singulières (première section) et leurs variations de puissance (seconde section) sont analysées.

À travers une interrogation sur la fonction ontologique des « degrés de réalité », et plus particulièrement sur les raisons du détachement progressif de Spinoza vis-à- 
vis de ce concept, C. Ramond met en lumière tout d'abord l'équivocité du terme de perfection. Il montre, par exemple, que dans l'expression « degrés de réalité ou de perfection ", le terme de perfection n'est pas redondant, mais qu'il permet - discrètement - de quantifier la réalité : en effet, « perfection » est employé pour désigner qualitativement «la perfection », c'est-à-dire « la réalité »; mais il est aussi employé pour désigner quantitativement « les perfections », c'est-à-dire les propriétés, neutres ou valorisées, d'une chose. Avec ce concept, on peut donc passer, notamment dans les premiers écrits, de la qualité (pensée selon le même et l'autre) à la quantité (pensée selon le plus et le moins). Une telle équivocité rejaillit dès lors sur la définition de l'être : celui-ci peut à la fois être d'une seule et même qualité, et se concevoir selon une multiplicité de degrés (ainsi l'« Être de Raison » est à la fois Être en tant que mode de penser, et Néant relativement à ce qui est signifié).

L'Éthique marque l'abandon progressif des « degrés de perfection ». Ce concept ne figure plus ni dans les propositions, ni dans les démonstrations (dans son analyse des preuves ontologiques, $\mathrm{C}$. Ramond montre qu'un nouveau concept est convoqué pour démontrer quantitativement l'existence de Dieu : la puissance); mais on le retrouve dans les appendices, préfaces et scolies qui jouent sur les sens qualitatif et quantitatif de la perfection - la conception quantitative de la perfection pouvant être soit intensive, sans multiplicité numérique, soit extensive, plurielle.

Avec l'analyse de la Lettre 12 à Louis Meyer, et dans le cadre d'une discussion permanente des thèses de Gueroult, commence une étude de la nature de l'infini. Le problème posé est le suivant : comment concilier la multiplicité des attributs et leur unité en Dieu? La distinction entre attributs est-elle réelle, et donc numérique, ou bien seulement modale? C. Ramond réfute d'abord l'idée de «distinction formelle », empruntée à Dun Scot, et utilisée par Deleuze, pour penser à la fois la diversité des objets considérés et l'unité de leur tout (multiplicité non numérique): ou la distinction est réelle, et l'unité fictive; ou l'unité est réelle, et la distinction fictive. En soulignant l'insuffisance du modèle mathématique pour résoudre le problème de la constitution de l'infini par lui-même, C. Ramond montre qu'il y a en fait une double infinité des attributs : une infinité qualitative et une infinité quantitative. L'infini qualitatif est enveloppé par le fini, sous la forme de l'indivisible (par exemple, l'étendue est en chacune de ses «parties » toujours qualitativement de l'étendue); et l'infini quantitatif contient le fini, sous la forme de l'indéfini (ainsi, dans la définition génétique de l'idée d'infini, l'indéfini est introduit par itération sans fin du fini). C. Ramond, par ailleurs, montre le statut ambigu de l'infinité des « modes infinis », qui est plutôt une « indéfinité » et met en valeur cette double tension qui caractérise la nature de l'infini : l'infini est finitisé lorsqu'il est pensé quantitativement; et le fini est infinitisé lorsque l'infini est conçu qualitativement, au risque cependant d'en faire un infini seulement potentiel.

Dans son étude des rapports entre qualité et quantité dans les choses singulières, C. Ramond met en avant le renversement de perspective opéré par Spinoza : celui-ci s'est efforcé de construire un Dieu sans quantité; il s'efforce désormais d'expulser la qualité «occulte » des modes finis, au profit de la quantité, c'est-à-dire d'une recherche de l'intelligibilité intégrale des choses.

Est analysée tout d'abord l'entreprise spinoziste de quantification de l'essence. Un problème se pose : comment la mathématisation de l'essence pourra-t-elle rendre compte du changement qui caractérise les choses singulières existant dans la 
durée? Ce souci de l'intégration du dynamisme des choses singulières dans leur essence est marqué chez Spinoza par la reprise, dans le champ de la nature naturée, d'une notion élaborée pour la nature naturante : à savoir l'idée d' " essence active ", identique à celle de "puissance ». Mais nous retrouvons une nouvelle fois la superposition des points de vue qualitatif et quantitatif : que l'essence soit caractérisée comme « rapport précis de mouvement et de repos » ou comme « effort pour persévérer dans l'être », la puissance (ou quantité) ne peut tenir lieu d'essence qu'à condition d'être précisément déterminée; et cette seule précision de la quantité la cristallise en qualité (une quantité précise n'étant pas la même qu'une autre). Comment, dès lors, penser la possibilité d'une variation de la puissance d'agir dans un même individu? Si l'essence est déterminée comme quantum de puissance d'agir, ou comme un rapport précis, alors toute variation quantitative lui est refusée; pour sauver la possibilité d'une telle variation, il faut donc disjoindre en quelque manière l'essence et la puissance. C. Ramond montre alors que la solution ne peut se trouver dans le recours au concept de "grandeurs intensives », dans lequel la qualité est encore déterminée par la quantité, puisque l'intensité n'est que l'introduction de la cause (quantitative, étendue) dans l'effet (qualitatif, inétendu).

C. Ramond est donc conduit à analyser le problème de l'individuation chez Spinoza. On retrouve ici, à l'échelle des modes, la question, rencontrée à propos de l'infinité des attributs et de la «distinction formelle», de la conciliation d'unités réellement distinctes (les individus-parties) en une unité capable de les intégrer (des individus-touts jusqu'à l' « individu total »). Pour résoudre ce problème, C. Ramond refuse l'idée, développée par Gueroult et Deleuze, selon laquelle l'essence serait située entre un minimum et un maximum (ce qui rendrait concevable la permanence de l'identité individuelle à travers des changements); il préfêre à cette idée d'une fourchette quantitative le modèle du pendule, qui peut être animé d'un nombre de mouvements d'autant plus grand qu'il est composé d'autres pendules, tout en observant une période qui correspond à une loi unique et précisément quantifiable. Cependant, C. Ramond reconnaît que Spinoza, pour maintenir l'unité qualitative de l'individu, a dû reculer sur la précision quantitative du rapport qui en constitue l'essence.

Afin, dès lors, de penser la légitimité des ruptures qualitatives dans la nature naturée, la notion d' « ordre commun de la nature " est examinée : cet ordre est à la fois cause de la connaissance inadéquate (qui perçoit des "conformités » et des "différences » illusoires) et objet de la connaissance adéquate (qui en perçoit de réelles, puisqu'il existe un ordre réel de la nature). Par conséquent, si l'infinité quantitative de la productivité divine rend légitime une infinité de compositions possibles des choses singulières (et oblige le philosophe à refuser l'étonnement), l'existence d'un ordre de la nature limite cependant les compositions admissibles : d'où l'affirmation d'une double discontinuité qualitative, reposant d'une part sur des différences essentielles entre choses singulières, et d'autre part sur des différences spécifiques - exigeant elles-mêmes l'existence de similitudes objectives entre individus. Le problème dès lors se pose de savoir qui peut légitimement constater ces similitudes.

Les dernières analyses de $\mathrm{C}$. Ramond sont donc consacrées aux modes de connaissance de la qualité. Il est montré en substance que le deuxième et le troisième genre de connaissance sont des connaissances de la qualité, soit commune à plusieurs choses singulières, soit propre à l'invariant de telle ou telle chose singu- 
lière. La science intuitive connaît ainsi les «essences des choses", qui sont les essences spécifiques des choses singulières. Ici s'affirmerait l'hyperqualitativisme de Spinoza : les seules réalités concevables sont les essences spécifiques, c'est-àdire des réalités générales et non singulières (car le singulier comme tout de parties et partie de touts s'efface dans la nature naturée). $C$. Ramond dès lors achève son analyse par l'examen des aspects qualitatifs de la théorie des passions dans L'Éthique et repose, en écho à Matheron, la question de l'existence de similitudes objectives.

Tout au long de son ouvrage, C. Ramond aura donc montré l'interpénétration des points de vue de la quantité et de la qualité autant dans la nature naturante que dans la nature naturée: son travail vaut avant tout par le constant souci de ne pas résoudre à tout prix les problèmes qui se posent. De nombreuses questions, sur lesquelles viennent buter bien des commentateurs, sont ainsi abordées, au premier rang desquelles se trouve la différence entre les «qualités occultes» et les "qualités manifestes » : question chez Spinoza non résolue, et qui pose le problème de la décision subjective - car, conclut C. Ramond, toutes les qualités sans exception sont toujours décidées.

Pascal SÉvÉRAC

Christiane Hubert, Les Premières Réfutations de Spinoza. Aubert de Versé, Wittich, Lamy. Paris, Presses de l'université de Paris-Sorbonne, 1994. 14,5 × 21, 150 p., bibliogr. («Groupe de recherches spinozistes. Travaux et documents ", 5).

Le groupe de recherches spinozistes de la Sorbonne s'intéresse aux débats d'idées qui ont précédé, accompagné ou suivi la publication des œuvres de Spinoza. Le cahier publié par Christiane Hubert étudie les réfutations de Spinoza dues à des apologistes chrétiens. L'un d'eux, Aubert de Versé, qui écrit L'Impie convaincu (1684), est persuadé qu'il est impossible de répondre à Spinoza sans concevoir deux principes éternellement coexistants, Dieu et la matière. Les deux autres, le bénédictin François Lamy, auteur du Nouvel Athéisme renversé (1696) et le théologien protestant Wittich, opposent à Spinoza la métaphysique cartésienne, correctement comprise, pour éviter que Descartes ne soit tenu pour responsable des détestables erreurs spinozistes. Dans ce débat, se lit clairement l'enjeu théologique. Les apologistes chrétiens partisans du cartésianisme se saisissent volontiers d'une philosophie qui laisse de côté toute preuve cosmologique de l'existence de Dieu, celle-ci leur paraissant liée à une philosophie naturelle dépassée. Les adversaires du cartésianisme avancent que la nature risque fort d'être absorbée en Dieu, puisque privée de tout dynamisme interne, elle a besoin de son impulsion à tout moment. Sous leur aspect technique, ces débats illustrent l'importance fondamentale du concept de création dans les théologies juive et chrétienne. Il implique la radicale séparation du Créateur et de la créature et c'est cette pensée de la séparation qui irritera les penseurs du $\mathrm{XIX}^{\mathrm{e}}$ siècle, tel Renan, qui revendiqueront l'unité des lois de la nature et de l'histoire. 
Paolo Cristofolini, Vico et l'Histoire. Paris, Presses universitaires de France, 1995. $11,5 \times 17,5,126$ p. («Philosophies », 58).

Vico n'est pas, ou plus, un inconnu en France; son œuvre bénéficie d'un intérêt croissant, dont témoignent plusieurs rééditions récentes en format de poche: la Science nouvelle relative à la nature commune des nations (Paris, Gallimard (« Tel »), 1992; version de 1744, et non de 1725, comme l'annonce faussement la couverture); De l'antique sagesse de l'Italie (Paris, GF-Flammarion, 1992), qui viennent compléter les traductions d'Alain Pons (Vie de Vico écrite par lui-même, Paris, Grasset, 1981). Si les écrits du philosophe napolitain sont ainsi devenus plus accessibles au lecteur de langue française, sa pensée n'en reste pas moins d'un abord difficile. Depuis les travaux de Jules Chaix-Ruy, qui avait œuvré, dans la première moitié du siècle, à la faire connaître, manquait un ouvrage d'introduction à cette pensée riche et dense, qui, par le style très singulier dans lequel elle s'exprime, en a rebuté plus d'un.

Le petit livre de P. Cristofolini, Vico et l'Histoire, vient combler ce manque d'une façon tout à fait remarquable. Suivant le principe de la collection dans laquelle il est publié, il est de dimensions restreintes et vise à une exposition claire et pédagogique des thèses de l'auteur. Le mérite de sa réussite, dans le cas de Vico, est d'autant plus grand qu'il est facile de simplifier, de schématiser sa pensée (comme cela a été fait : qui ne connaît Vico comme le père de la « loi des corsi et ricorsi " et... c'est tout!) et difficile d'y introduire sans rien céder de son intime complexité.

L'auteur aborde frontalement la pensée de Vico, en prenant pour objet le problème central qui l'anime : celui de l'histoire. Si Vico affirme être le conquérant d'une terre nouvelle, que les philosophes n'ont jamais foulée, préférant à l'étude du "monde civil des nations » celle de la nature, P. Cristofolini retrace les étapes de cette conquête et met d'abord en place « les matériaux » (chap. 1), puis expose la " théorie de l'histoire » (chap. 2) qui s'est dégagée de leur agencement progressif, alliant ainsi, selon une méthode toute vichienne, philologie et philosophie.

Or il faut entrer dans le détail de l'histoire des textes vichiens si l'on veut pénétrer la Science nouvelle qui en est la dernière expression. P. Cristofolini explique très bien comment, chez Vico, la distinction entre trois âges de l'humanité - "âge des dieux " (chap. 3), « âge des héros » (chap. 4), « âge des hommes » (chap. 5) -, dans son apparente simplicité, recouvre une théorie complexe de l'histoire, dont le problème central, quoique seulement latent dans la dernière version de la Science nouvelle (1744), est celui de la délimitation des domaines respectifs de l'histoire sacrée et de l'histoire profane; l'herméneutique vichienne ne se constitue qu'en définissant rigoureusement son champ d'investigation, les « origines obscures » de l'humanité relevant de l'histoire sacrée dont la Bible a conservé le témoignage, tandis que ses «origines fabuleuses » deviennent lisibles à travers une interprétation originale des mythes, qui nous livre la clef de l'histoire profane. Il importe, dès lors, d'être au fait des débats que Vico engage avec les autorités ecclésiastiques et de la singularité de sa version de la Genèse, de la Chute, du déluge universel et de la confusion des langues. En exposant avec clarté ces présupposés religieux et métaphysiques, P. Cristofolini est à même de poser les questions les plus radicales 
que soulève la pensée de Vico — « les hommes font-ils leur histoire? » (p. 26); quel est le rôle de la Providence? - et de proposer sa propre compréhension du problème (dans la conclusion sur «l'animisme de Vico »).

Enfin, le moindre des mérites de ce livre n'est pas de publier, suivant le principe de la collection, une série de textes de Vico et de les donner à lire dans la traduction de Michelet qui, si elle est oubliée, faute d'une réédition récente, reste intéressante et riche d'enseignements.

Anne-Sophie MENASSEYRE

Lectures de Montesquieu. Actes du colloque de Wolfenbüttel, 26-28 oct. 1989, réunis par Edgar Mass et Alberto Postigliola, prés. par E. Mass, préf. de Jean Ehrard. Naples/Paris/Oxford, Liguori Ed./Universitas/Voltaire Foundation, 1993. $19 \times 24,5,184$ p. (« Cahiers Montesquieu », 1).

Cet ouvrage contient les actes du premier colloque de la société Montesquieu qui s'est tenu dans la Herzog August Bibliothek à Wolfenbüttel. Tentons un regroupement, simplificateur certes, mais selon nous nécessaire, des treize communications qu'il réunit.

Un premier groupe de deux articles retrace la réception de l'Esprit des lois en France (Louis Desgraves) et celle de l'œuvre de Montesquieu en Allemagne (Frank Herdmann).

L. Desgraves établit qu'aux premiers compliments des lecteurs attentifs que furent Cerati, Hume ou Hénault, succédèrent bientôt les attaques des financiers, puis des religieux. Dès 1750 , la situation se dégradait, jusqu'à la mise à l'index de novembre 1751.

Selon F. Herdmann, Montesquieu a été abondamment lu, commenté, controversé en Allemagne : entre autres, Frédéric II s'en inspire, l'économiste von Justi refuse la théorie des climats et les membres de l'académie de Berlin blâment Montesquieu pour sa connaissance insuffisante de l'histoire, son irréligion et l'absence de système et de doctrine générale.

Un deuxième groupe traite de philologie et d'esthétique avec trois articles d'Artur Greive, de Kai-Ulrich Hartwich et de Peter-Eckhard Knabe.

Le premier montre que Montesquieu n'a pas consacré de traité important aux questions de philologie. Mais des propos épars indiquent clairement ses préoccupations concernant la langue, en particulier l'ambiguïté des mots, lesquels égarent la pensée : il faut donc éviter ce défaut dans la composition des lois. Montesquieu accorde également une grande attention à la traduction, laquelle ne peut être qu' " une belle infidèle ".

Les deux autres articles sont consacrés aux problèmes posés par la notion de goût. K.-U. Hartwich tente de montrer que, pour Montesquieu comme pour Freud, les processus psychiques sont liés à des problèmes d'économie de l'âme comme prin- 
cipe régulateur entre des « instances » dont le plaisir est le principe moteur. Selon les deux penseurs, les sentiments n'ont pas dans notre âme une cause unique. Mais K.-U. Hartwich souligne que cette comparaison a des limites : l'âme est transparente pour Montesquieu et n'offre pas la moindre trace d'inconscient!

Le troisième article relate les difficultés rencontrées par les philosophes et encyclopédistes du xviri ${ }^{e}$ siècle pour transposer la notion de goût dans la théorie de l'art. Cet historique permet de tracer une frontière entre rationalisme et sensualisme. Montesquieu, dans sa contribution à l'Encyclopédie sur cette question, se range du côté des sensualistes. Le sentiment est, pour lui, le point de départ du jugement esthétique, ce qui réduit l'analyse du goût à celle des plaisirs. D'Alembert puis Kant opéreront la synthèse des deux grandes philosophies du siècle.

Un troisième groupe, constitué par les articles de Lorenzo Bianchi, de Claude Lauriol et de Salvatore Rotta, se consacre aux rapports de Montesquieu avec la religion.

S. Rotta livre une analyse très érudite sur les sources des études de Montesquieu concernant le paganisme. L'attitude du Président est favorable à la « religion des païens ». Son interprétation personnelle des Satyres et des Silènes est en rapport avec l'histoire de l'anthropologie : il s'agit de la question alors importante des dieux animaux assimilés à des singes. De plus, pour Montesquieu, l'idolâtrie est indestructible, inhérente à la nature humaine, et le paganisme est une forme de religion tolérante et non répressive, ce qui permet de critiquer implicitement l'aspect contraire propre au christianisme.

C. Lauriol analyse, dans cette direction, la lecture que fait Court de Gébelin de Montesquieu. Lecture originale : ce pasteur protestant voit dans l'auteur de l'Esprit des lois un ardent défenseur de la religion réformée : il veut montrer que le protestantisme n'est lié ni au républicanisme ni au désordre. Au contraire, en tirant à lui, de manière un peu forcée, les exemples du Président, Gébelin montre que ce dernier voit dans le protestantisme la religion de la liberté et de l'avenir. Une lecture inspirée par l'affaire Calas et qui vise l'intransigeance catholique.

Enfin, L. Bianchi démontre, à partir de la Dissertation sur la politique des Romains dans la religion, que Montesquieu prône la nécessité politique de la religion et surtout la tolérance romaine qui fonda un véritable syncrétisme au service de l'État. Il s'agit du même argument contre le christianisme, et les communications de ce groupe aboutissent à des conclusions analogues.

Le quatrième et dernier groupe touche aux préoccupations majeures de Montesquieu: l'histoire et la politique, avec les articles de Georges Benrekassa, C. P. Courtney, Domenico Felice, Alberto Postigliola et Françoise Weil.

À partir du problème du droit naturel subjectif, $G$. Benrekassa tente une confrontation des idées de Montesquieu et de celles de Kant. Il est trop facile d'opposer l'universalité de la loi générale de la liberté qui, selon Kant, définit a priori les libertés de chacun, au relativisme des lois chez Montesquieu. Pour ce dernier, une doctrine universelle du droit n'est certes pas une évidence. Mais G. Benrekassa montre qu'il y accord entre les deux penseurs sur le fait qu'il n'y a pas d'ordre juridique qui tienne sans lien accepté, d'ordre éthique, de l'individu avec le pacte social. Si Montesquieu laisse place à une « heureuse contingence» des droits dans leur réalité historique, il ne s'en interroge pas moins, avec Kant, sur la relation qui 
doit exister entre l'individu et le citoyen. Pour les deux philosophes, ce n'est jamais l'individu qui possède un droit : c'est l'espèce humaine qui est en cause. Et G. Benrekassa conclut qu'il y a bien, chez le prétendu positiviste Montesquieu, une référence claire à la légitimité philosophique d'un droit abstrait.

A. Postigliola se demande si L'Histoire véritable ne pourrait pas être un «prélude épistémologique " à l'Esprit des lois. Après une étude érudite de la chronologie et des sources de L'Histoire véritable - authentique conte philosophique pour Montesquieu - l'auteur montre que le Président, en moraliste, y illustre la faillite de l'anthropologie et de la science politique du Grand Siècle qui n'avait pas répondu à l'interrogation de Pascal sur la relativité du droit et de la justice. La diversité des mœurs s'explique, à travers la fable de la métempsycose, par l'identité qui en résulte enfin: ce sera la démarche même de l'Esprit des lois, un voyage dans l'essence même de l'humanité. L'auteur aboutit a une conclusion similaire à celle du précédent article, à savoir la cohérence du dessein de Montesquieu.

Les trois autres articles s'intéressent, comme dans notre premier groupe, aux problèmes posés par la réception de l'œuvre du Président, mais plus spécifiquement ici du point de vue de l'histoire et du droit.

D. Felice décrit l'influence de Montesquieu sur deux philosophes italiens de la première moitié du XIX ${ }^{e}$ siècle. Vincenzo Cuoco commente et loue la leçon de relativisme de Montesquieu, qui complète celle donnée par Vico, sa thèse de la supériorité des mœurs sur les lois, et celle du "ce n'est pas la fortune qui domine le monde » des Considérations qui trouve son écho dans la providenzialità della storia du penseur italien. Mais $V$. Cuoco ne ménage pas ses critiques : la vérité absolue du droit manque de fondement et Montesquieu de méthode. Pour Gian Domenico Romagnosi, la critique négative prévaut sur trois points : la conception de la loi d'abord (selon le penseur italien, la loi est le résultat de divers rapports et non ellemême un rapport), la théorie des formes de gouvernement ensuite (Montesquieu se voit reprocher de privilégier le fait sur le droit), le principe de la division des pouvoirs enfin (c'est la polémique la plus âpre, avec une critique de la constitution anglaise si prisée par le Président).

C. P. Courtney, quant à lui, s'interroge sur le sort des idées de Montesquieu pendant la Révolution: que pensait Montesquieu de la révolution et qu'a pensé la Révolution de lui? L'auteur commence par la seconde question et montre que Montesquieu le traditionaliste a été opposé à Rousseau le révolutionnaire et est devenu l'auteur favori de l'opposition nobiliaire. Burke s'est en effet inspiré de Montesquieu dans sa critique de la Révolution. Pour répondre à la seconde question, l'auteur nuance fortement la thèse d'un Montesquieu qui n'aurait pas été surpris par la Révolution. Les vues du Président sur une réforme politique restent modérées. En ce qui concerne la notion de révolution, quand il ne l'emploie pas au sens de « restauration ", Montesquieu pense que la monarchie, avec ses pouvoirs intermédiaires, semble particulièrement bien équipée pour éviter ce genre de changement brusque. On ne trouve donc pas de théorie de la « révolution violente » chez Montesquieu : selon l'auteur, le Président n'aurait guère approuvé la Révolution française.

Enfin, appliquant les propositions précédentes à un cas particulier, F. Weil s'interroge sur la concordance des idées de Vivant Carion, unique rédacteur du Journal de la Côte d'or, avec celles de Montesquieu : V. Carion tire son républicanisme modéré des idées du Président, et ce aussi bien contre l'extrémisme de la Ter- 
reur qu'en faveur d'un retour à l'ordre propre au Directoire. L'auteur arrête son examen au début de l'Empire napoléonien.

Ce volume, remarquable dans sa présentation matérielle, a le mérite de donner des lectures européennes et croisées de l'œuvre de Montesquieu dans toute son ampleur. En dépit de l'absence, que l'on peut regretter, d'un classement perceptible des communications, chaque lecteur y pourra trouver une vue neuve ou l'approfondissement de ses propres intérêts et recherches.

Jean-Marc RoHRBASSER

Rousseau, l'Émile et la Révolution. Actes du colloque international de Montmorency, 27 sept.-4 nov. 1989, publ. par Robert THIÉRY. Paris, Universitas, 1992. 17,8 × 24,2, xii-559 p., 32 ill., index.

L'enjeu de ce colloque est énoncé par Robert Thiéry : redéfinir Émile, établir son importance par rapport aux événements révolutionnaires. L'intérêt de la Convention pour les manuscrits de Rousseau, souligné par J. Sgard, atteste la pertinence de ce travail. L'ensemble des interventions réunies dans ce volume, très variées et forcément inégales, s'organise en quatre moments principaux.

C'est bien évidemment le problème général du rapport de la pratique politique et révolutionnaire à la pensée pédagogique de Rousseau qui constitue l'essentiel du recueil. On doit à Rousseau de comprendre que «la liberté n'existe pas hors de ma liberté » (M. Soëtard) : c'est pourquoi la volonté générale, « regard global sur l'ensemble des volontés particulières » (G. Lafrance) s'éprouve aussi en chacun de nous, dans le silence des passions. Cette inspiration, ainsi que le thème des vertus antiques, pénètrent indéniablement les discours révolutionnaires; on objectera néanmoins à l'auteur qu'il est difficile de trouver en Rousseau le défenseur d'une séparation des pouvoirs (au sens d'une théorie des parties de la souveraineté) qui, chez ce philosophe, ne se déduit pas directement de la nécessité de contrôler l'exercice de l'autorité. Quoi qu'il en soit, l'exigence d'un nouvel ordre exprimée dans l'Émile est proprement morale, et s'impose immédiatement dans le sentiment - J. Domenech propose à cet égard une comparaison très utile avec Diderot, et montre qu'avant Hegel, c'est l'apologiste Barruel qui pose la question de l'efficacité pratique de cette morale du sentiment. Mais la réception de l'œuvre rousseauiste ne se concentre pas sur ce seul problème. Le rejet de la littérature affiché par Rousseau, s'il se retrouve dans de nombreux ouvrages pédagogiques de l'époque, comporte cependant deux originalités majeures selon S. Menant: les livres et le théâtre, dans la mesure où ils interviennent dans la formation de la sensibilité, peuvent contribuer à l'affirmation du bonheur; de plus, Rousseau écarte toute finalité pratique de la formation littéraire, puisque Émile sera artisan - mais de ce point de vue, il est utile de rappeler que, si Rousseau destine les dictionnaires aux " égouts de la littérature », le précepteur a bien lu les descriptions de métiers fournies par l'Encyclopédie, dont l'élève prendra plus tard connaissance. Dans une belle étude des figures du peuple représentées dans l'Émile, L. Viglieno rappelle ainsi 
comment Émile contribue, parmi les paysans, au perfectionnement des techniques agricoles : c'est le seul enseignement dont le peuple peut avoir besoin, et nous notons qu'il correspond bien à cet accouchement des savoirs particularisés dont parle Diderot dans le Prospectus de l'Encyclopédie. Ce n'est donc pas sans raison que des lecteurs avisés comptent Rousseau au nombre des «philosophes », malgré les thèmes chrétiens qui s'affirment dès le premier Discours - avec L. A. Russell, on s'intéressera aux principaux rédacteurs du Journal de Trévoux, Berthier et de Jolivet. De là, il ne faut certainement pas dessiner, avec J. Oërlemans, le portrait d'un Rousseau aigri qui n'aurait pas eu la carrière qu'il rêvait : les meilleures descriptions de l'ambition se trouvent d'ailleurs sous la plume de l'auteur des Confessions. J.-J. Tatin-Gourier manifeste encore le caractère subversif, i. e., dans les termes de l'époque, "philosophique » de la pensée de Rousseau, en signalant l'occultation prudente de toute critique du système monarchique dans les recueils de fragments parus avant 1789 : Émile est avant tout lu comme un traité d'éducation, bien qu'il soit subordonné aux fins politiques fixées dans Le Contrat social. La précieuse synthèse proposée par $B$. de Negroni enrichit notablement la comparaison de ces textes. L'auteur souligne les emprunts que le législateur et le précepteur opèrent dans leurs lexiques respectifs, et évalue ensuite la compatibilité des thèses sur l'éducation avancées dans l'Émile et dans les Considérations sur le gouvernement de Pologne : à terme, l'une et l'autre figure doivent faire passer le peuple ou l'enfant du monde des relations de nécessité à celui des relations d'obligation — on restera plus réservé sur l'interprétation du rôle de la religion, comprise comme analogue politique des stratagèmes du précepteur. Mais cette étude permet d'éviter certains malentendus : au lieu de tenir avec L. G. Crocker qu'Émile peut faire tout ce qu'il veut, pourvu qu'il réalise " ce que le maître veut qu'il veuille », on comprendra que l'enfant doit faire ce que le précepteur sait qu'il peut - selon nous, la «duplicité » est ici du même type que celle qui caractérise le législateur dans Le Contrat social, II, 6. L'étude de G. A. Roggerone dégage ensuite l'originalité de la conception rousseauiste de la révolution : il montre en particulier comment Rousseau associe, à la différence de Montesquieu, l'idée d'une guerre civile, forcément catastrophique, au sens astronomique habituel, qui désigne une régénération. Néanmoins, la vocation d'Émile à n'obtenir que la conversion des classes privilégiées aux vrais principes du droit est contestable. Certes, Rousseau souhaite éviter à son élève le poids des grands bouleversements politiques qui sont du reste inenvisageables en France : il se pose alors en Socrate moderne (M. B. Ellis). Mais son rôle dans la constitution d'une sensibilité révolutionnaire est justement apprécié par Jung-Ja Moon. Signalons, d'autre part, l'étude très stimulante de K. Kamai qui, après avoir rappelé comment Rousseau renouvelle la question de l'origine du mal, met en évidence le cas particulier que constitue l'injustice sociale : cette anomalie insupportable requiert bien l'intervention de la Providence, qui rétablit l'ordre pour l'âme immortelle. L'antinomie de l'homme et du citoyen peut donc se résoudre au niveau religieux Robespierre, par le culte de l'Être suprême, voudra ainsi célébrer l'intégration réussie de l'individu dans sa fonction politique (J. Boulade-Ayoub). Dans leur lecture des textes de Rousseau, les hommes de la Révolution ne pourront donc pas ignorer le Vicaire savoyard, même s'ils éviteront parfois, comme Robespierre, de mentionner son caractère chrétien - en témoigne l'étude instructive fournie par Y. Touchefeu. 
La deuxième partie des actes du colloque de Montmorency aborde le rôle de la femme dans l'Émile et, plus généralement, la fonction de la famille dans le système politique de Rousseau. On signalera l'étude originale de M. Morgenstern consacrée au rapport de la révolution avec la vie quotidienne dans l'œuvre de Rousseau; elle comporte en particulier une analyse éclairante de la qualité de l'assentiment que Sophie peut donner à la vertu. Comme l'indique plus tard M. O'Dea, dans un texte très pertinent, la compagne d'Émile ne doit pas premièrement dire la vérité, mais bien prévoir ce qui convient le mieux à son époux. D'une façon plus générale, ces articles montrent bien que les structures profondes de la vie privée sont pourvues de significations politiques. Plusieurs textes permettent, d'autre part, de lire Rousseau en son temps, sur ces questions. La contribution de I. Brouard-Arends situe les thèses de l'Émile par rapport aux récits féminins de la maternité au xviII siècle (chez Madame d'Épinay, Madame de Charrière et Madame de Genlis); E. Badinter oppose au modèle rousseauiste de la différence des sexes, qui triomphe au XIX ${ }^{e}$ siècle, l'unification de l'espèce humaine prônée par Condorcet. Enfin, A. Leclerc rappelle opportunément, contre des accusations parfois superficielles, que le plan de Rousseau était aussi bien d'offrir aux femmes la meilleure éducation possible, afin de fonder le pacte conjugal comme personne morale.

La troisième partie de ce volume concerne la présence de Rousseau dans la Révolution française, d'abord d'un point de vue «matériel », à travers les éditions de l'Émile (J.-A.-E. McEachern). Le fait que cet ouvrage soit présenté comme un roman conduit T. Marshall à souligner le rôle de l'imagination dans les entreprises pédagogique et politique : cette faculté commanderait tout le domaine pratique, parce qu'elle frappe vivement le sentiment. Mais, dans cette théorie du sentiment à laquelle se reconduiraient les fondements du politique, l'auteur ne souligne pas assez clairement que, selon Rousseau, la conscience n'acquiesce jamais qu'au bien que la raison lui présente. $R$. Granderoute propose un tableau général très précieux de la présence de l'Émile au sein des discours sur l'éducation jusqu'en 1795 : ce sont les résistances de la pédagogie rousseauiste qui apparaissent ici, autant que ce qu'elle apporte effectivement aux convocations très diverses des révolutionnaires (J. Bloch). L'enseignement de la lecture, que veut promouvoir la Convention (N. Senior), de même que les tentatives des pédagogues «professionnels » (Serane, étudié par P. Jimack) témoignent de la variété des choix qui s'opèrent dans la référence au traité de 1762 . Il faut surtout mentionner deux articles importants: G. Incorvati, d'une part, montre de quelle façon l'universalité du travail comme valeur, affirmée par Rousseau, va constituer un problème pour les révolutionnaires - tandis qu'en 1791, la nation s'identifie aux propriétaires, la Convention va découvrir que l'égalité politique ne suffit pas pour aborder les revendications économiques. D'autre part, C. Larrère établit bien, à partir d'une étude précise des textes de Babeuf, que ce sont les révolutionnaires (puis, dans le discours philosophique, Fichte) qui vont réaliser directement la liaison du pédagogique au politique, que Rousseau ne concevait pas sans la médiation de l'autonomie individuelle. Sans entrer dans le détail de cette étude, signalons au moins qu'elle découvre dans l'éloge du travail artisanal le véritable point d'accord de Rousseau et Babeuf. Deux autres interprétations significatives sont enfin abordées : celle de Mercier, qui présente l'intérêt de lire l'Émile sous l'angle de la régénération (O.-H. Bonnerot), et celle de 
Barruel, qui déplore le ralliement passager mais irrécupérable de Rousseau, le " sophiste de l'impiété », à la secte des encyclopédistes (C. Porset).

La dernière partie de ce recueil présente quelques aspects de la fortune littéraire et philosophique d'Émile. Y. Chastagnaret présente l'Émile du xIX siècle d'Esquiros, dont on s'amuse de constater qu'à la différence de son modèle, il s'agit d'un ouvrage qui se finit bien. La diffusion des thèses rousseauistes en Pologne, mais aussi en Hongrie et en Chine, est également évoquée - on retiendra que les révolutionnaires hongrois avaient parfaitement identifié ce qui, chez Rousseau, se comprenait dans l'esprit de la Réforme protestante. A. Delamare expose les résultats d'une étude lexicographique du rapport entre les textes de Rousseau et les Déclarations des Droits de l'Homme en France, qui illustre le rôle du vocabulaire dans la formation des idées politiques. Les actes de ce colloque incluent en outre quatre articles consacrés à la musique. Signalons le travail de $\mathrm{S}$. Kleinman, qui défend vigoureusement la méthode d'enseignement du système des intervalles préconisée par Rousseau. Dans une autre perspective, D. Paquette circonscrit l'influence de Rousseau sur la musique révolutionnaire, à travers les hommages musicaux qui sont destinés à l'auteur du Devin du village, et dans l'utilisation des airs les plus connus de cet opéra-comique.

André CharraK

Michel Meyer, Science et Métaphysique chez Kant. Paris, Presses universitaires de France, 1995. 12,5 × 19, 248 p., bibliogr. («Quadrige», 193).

M. Meyer voit dans l'œuvre de Kant le travail d'une double question : faut-il renoncer à la métaphysique parce que l'entendement n'a pas de sens au-delà de la sensibilité ou faut-il à l'inverse concevoir ces limites assignées à l'entendement, comme ce qui permet, par leurs restrictions mêmes, de laisser libre et possible un champ propre de la raison? Là réside tout le paradoxe de la Critique : si l'objet à connaître est le même que l'objet donné à la sensibilité, l'entendement est inutile à sa constitution; mais si ce n'est pas le même objet, l'objectivation issue de l'entendement ne peut plus coïncider avec l'objet du sensible.

C'est à la lumière de ce paradoxe que $\mathrm{M}$. Meyer tente d'examiner la différence entre les deux éditions de la Critique de la raison pure. À chacune des branches du paradoxe correspondrait soit la première, soit la seconde version de la Critique, la Déduction transcendantale étant à la fois le choix opéré sur ce qu'est l'objet et la justification de ce choix. Si l'objet est donné, il faut démontrer néanmoins la nécessité du travail de l'entendement (ce sera le but de la version B); si l'objet n'est pas donné, il faut néanmoins montrer que la synthèse d'un objet de l'expérience a lieu, que l'entendement a valeur objective (version A).

Cette étude de la Critique de la raison pure s'inscrit dans le cadre plus large d'une réflexion sur l'ontologie, que l'auteur définit comme étant la négation de ce qui fut, avec Socrate et avant Platon, le discours de la philosophie, à savoir une interrogation; la question socratique portait ainsi plus sur le «qu'est-ce que $\mathrm{X}$ ? » (sans souci de la réponse), que sur la question de savoir «qu'est-ce (que) $\mathrm{X}$ ? ». 
Le glissement du premier au second type de questionnement marque précisément le passage de la philosophie interrogative (la problématicité irréductible de $\mathrm{X}$ ) à l'ontologie (l'interrogation sur l'être de X), celle-ci n'étant que la négation par la proposition, de cette interrogativité du philosopher. L'exclusion des alternatives qui caractérise le discours propositionnel se fonde ou plutôt s'abîme dans la dénégation d'une question inaugurale, à savoir : pourquoi fonder le discours sur la nécessité de l'exclusion des alternatives c'est-à-dire sur l'apodicticité ? Le refoulement (propositionnel) de cette question fait que l'apodicticité même se trouve sans fondement. En effet, si cette quête initiale d'un fondement propositionnel pouvait s'énoncer, il faudrait qu'elle s'abolisse comme quête, qu'elle soit déjà apodictique; il faudrait que la recherche du point de départ absolu fasse déjà partie de cet absolu.

Sans pour autant rejoindre Heidegger, qui selon lui, reste prisonnier de l'ontologie tout en prétendant la dépasser, M. Meyer montre que la question de l'être n'est pas l'impensé de la philosophie mais son impasse et qu'elle doit, à ce titre, être considérée comme une notion philosophique impossible. La question qui alors se pose est de savoir si une métaphysique est possible en dehors de l'ontologie.

Descartes comme Kant ont ainsi tenté de dissocier la métaphysique de l'ontologie en montrant que la science dit ce qui est, et que la tâche de la philosophie est désormais de fonder le discours scientifique comme discours $d u$ savoir. La métaphysique n'est-elle alors que cette impossibilité de l'ontologie (comment dire l'être si l'être se dit de plusieurs manières?) ou bien s'ouvre-t-elle sur d'autres exigences?

Chez Kant, il s'agit, d'une part, de limiter l'usage des principes de la raison à l'expérience si l'on veut atteindre la connaissance et, d'autre part, d'établir une positivité de la raison, qui ouvre sur la foi et l'action. Il faut restreindre les prétentions de la métaphysique à vouloir tout régir selon les canons de la science, comme si tout pouvait se réduire à l'objet empirique, au phénomène : en montrant que l'on ne peut réduire la pensée à la science, le métaphysique à l'empirique, le noumène au phénomène, Kant laisse ainsi le champ libre pour une métaphysique « autre ». Mais quel statut alors accorder à la métaphysique : est-elle une science ou doit-elle s'abolir comme connaissance pour n'être plus que raison pratique ou foi ? C'est à cette question que Science et Métaphysique chez Kant tente de répondre.

Kant a entrevu le caractère "problématologique » du discours philosophique (lorsqu'il montre, par exemple, que la Critique n'a pas affaire aux objets de la Raison mais à la Raison elle-même et aux problèmes qui naissent de sa nature propre) mais, comme le fit Descartes avant lui, il le nie par la suite, en acceptant pour présupposé et norme, l'apodicticité (et non l'interrogativité) et le propositionnalisme (et non le problématique). À l'inverse, il s'agirait de se maintenir dans cette irrésolution, cette interrogativité fondamentale de la philosophie sans succomber à la tentation d'une logique de la proposition (qu'elle soit celle de la non-contradiction, du Cogito comme fondement du savoir ou du transcendantal).

Attribuer un projet ontologique à Descartes ou à Kant, qui sont des penseurs de la proposition, n'est pas contradictoire puisque la fondation de l'ordre propositionnel est une nécessité née de l'ontologie (comme le prouve le principe aristotélicien de non-contradiction) mais une nécessité impossible à assumer car l'ontologie la condamne d'emblée, la dispersion de l'être étant insurmontable. Renoncer à l'ontologie permet alors seul de dire ce qui est : d'où l'accès indirect à l'être, par la ques- 
tion des conditions de son accessibilité, que prônent et réalisent Descartes comme Kant.

À partir de l'ambivalence fondamentale de l'entendement, à la fois nécessairement autonome (pour rendre possible la morale et les errances de la métaphysique dogmatique) et privé de sens sans l'apport de la sensibilité, M. Meyer propose une interprétation de l'ensemble de la Critique de la raison pure, même si l'on peut toutefois regretter que le lien entre métaphysique et morale ne soit pas suffisamment analysé. Le fil conducteur que l'auteur propose, à savoir le refoulement, par la logique de la proposition, de l'interrogativité ou de la problématicité intrinsèques au discours philosophique, tel que l'inaugure Socrate dans ses dialogues aporétiques, offre certes une lecture aussi nouvelle qu'éclairante de la Critique mais on peut néanmoins être en droit de se demander si elle n'est pas réductrice. En effet, est-ce seulement l'apodicticité que recherche Kant dans ce que l'auteur désigne comme un « refoulement » de l'interrogativité du philosopher? ou bien est-ce le pouvoir de la raison à se dépasser toujours elle-même et par elle-même, ce dépassement l'amenant à acquérir ce qui restait le privilège de la réalité sensible, à savoir la facticité; n'est-ce pas là le dernier mot de l'entreprise critique : la production de la raison comme un Fait?

Laurence Devillairs

Georg Wilhelm Friedrich Hegel, Phénoménologie de l'esprit. Prés., trad. et notes par Gwendoline JARCZYK et Pierre-Jean Labarrière. Paris, Gallimard, 1993. $14 \times 22,5,917$ p. ( « Bibliothèque de philosophie $)$.

La difficulté de compréhension de la Phénoménologie de l'esprit est proverbiale; mais la séduction exercée par ce chef d'œuvre de Hegel, tout particulièrement sur le public français, l'est aussi. Celui-ci devrait donc être reconnaissant à Gwendoline Jarczyk et à Pierre-Jean Labarrière de lui avoir donné de quoi faciliter la compréhension de l'œuvre - et de quoi nourrir son attrait. Car ce qui nous est offert ici n'est pas seulement une nouvelle traduction. La présentation substantielle, les nombreuses notes explicatives qui accompagnent chaque page, ainsi que plusieurs appendices - parmi lesquels on notera, tout particulièrement, un index détaillé des matières - tout cela fait de l'ouvrage présent un outil de travail extrêmement précieux non pas seulement pour l'étude de la Phénoménologie de l'esprit elle-même, mais aussi pour l'interprétation du système hégélien dans sa totalité.

La Phénoménologie de l'esprit est difficile à traduire, plus difficile encore que les autres ouvrages de Hegel. Dans ce livre, le premier qu'il publia, le philosophe d'léna ne se contente pas, en effet, d'user d'une terminologie philosophique courante à son époque, mais peu familière et même, souvent, totalement inconnue aujourd'hui. Il ne se contente pas de remettre cette terminologie en question et d'élaborer, chemin faisant, son propre vocabulaire. Comme le soulignent les traducteurs eux-mêmes, il puise aussi largement, bien plus largement encore que dans ses autres ouvrages, dans le vivier du langage ordinaire, dans le parler de son temps. La 
traduction de Jean-Pierre Lefebvre, publiée peu de temps avant la traduction présente, avait choisi d'exploiter ce filon. P.-J. Labarrière et G. Jarczyk ont, quant à eux, voulu mettre l'accent sur le caractère essentiellement philosophique de l'œuvre : ils déclarent dès l'abord que ce qu'ils entendent faire valoir dans leur traduction est non pas la forme du discours, mais l'« intention de sens ». Dans ce projet, ils restent jusqu'au bout conséquents avec eux-mêmes. Ils ne se sont permis aucune liberté dans leur travail austère de traducteurs : entendant respecter le rythme même de la phrase, sa ponctuation, et jusqu'à ses lourdeurs, ils reconnaissent n'avoir jamais coupé une phrase (sauf si c'était vraiment nécessaire, c'est-à-dire dans une dizaine de cas tout au plus), et cela quelle que soit la complexité ou même, parfois, le caractère incorrect de sa construction. Cette exigence de fidélité extrême garantit au lecteur une traduction conséquente des concepts hégéliens, réalisée selon le principe, scrupuleusement respecté, d'une correspondance biunivoque stricte entre l'allemand et le français. Certaines des grandes œuvres du système hégélien de la maturité - la Science de la logique (trad. Gwendoline Jarczyk et Pierre-Jean Labarrière, Paris, Aubier, 1972, 1976, 1981), l'Encyclopédie des sciences philosophiques (trad. Bernard Bourgeois, Paris, Vrin, 1970, 1988) - avaient déjà été traduites en français selon le même principe. Mais celui-ci n'avait pas encore été appliqué à la Phénoménologie de l'esprit, sans doute parce que souvent, trop souvent encore, tout particulièrement en France, cette œuvre est considérée comme antérieure au système, parfois même comme extérieure à lui. La tâche méritait d'être accomplie : les lecteurs français, si longtemps restés sous le charme de l'introduction à-la-Hyppolite, ou à-la-Kojève, devront bien maintenant se plonger dans le hégélianisme lui-même.

Ils découvriront ici que le texte, mais aussi - et peut-être surtout - certains des passages qu'ils connaissent le mieux sont, dans la traduction Jarczyk/Labarrière, méconnaissables. Le passage sur la «dialectique du maître et de l'esclave » tant célébré par Kojève est maintenant intitulé non plus « indépendance et dépendance de la conscience de soi », mais " autostance et inautostance de l'autoconscience ": il fallait bien, pour traduire le terme allemand de Selbstbewußtsein sans enfreindre le principe de correspondance biunivoque, chercher une autre expression que celle de "conscience de soi »; car celle-ci constitue la traduction littérale de l'expression allemande Bewußtsein von sich, que Hegel utilise aussi, quoique rarement. Ce fut donc la notion d' " autoconscience " qui fut choisie, ou plutôt constituée, sur le modèle d'autres expressions françaises : automouvement, autodétermination, etc. Et il convenait aussi de faire ressortir dans la traduction française le sens premier et commun en allemand de notions comme Selbständigkeit et Unselbständigkeit: « tenir debout » soi-même, ou non; d'où le recours à d'autres néologismes : « autostance » et « inautostance ». Les lecteurs de Hegel, comme d'ailleurs de tout autre philosophe, savent que la constitution d'un vocabulaire et, par-delà celui-ci, d'une langue en laquelle s'exprimer et s'entendre, fait intégralement partie du travail philosophique lui-même. En ce sens, l'espoir des traducteurs de voir leurs vocables peu à peu « agrégés au trésor lexical qui est le nôtre, bien souvent formé à la suite de telles audaces » (p. 55), est sans doute justifié. Des précédents comme, par exemple, la traduction du terme aufheben par le néologisme «sursumer » parleraient en faveur de telles solutions. 
Il est cependant permis de se demander si dans certains cas, comme dans celui de la distinction entre Selbstbewußtsein et Bewußtsein von sich, les raisons données suffisent à justifier ce que les traducteurs dénomment des « innovations lexicales » : n'aurait-il pas suffi de donner le terme allemand entre parenthèses, ou en note, dans les rares cas où Hegel utilise le vocable Bewußtsein von sich? Le problème de savoir comment distinguer en français entre la Geschichte, l'histoire effective, les événements historiques eux-mêmes (res gestae), et l'Historie ou narration de l'histoire effective, récit des événements (historia rerum gestarum), aurait pu, peut-être, être résolu de façon semblable plutôt que par l'utilisation, pour traduire le second terme (Historie-historisch), du français « historial » (p. 67, par ex.) : ce dernier néologisme est en effet déjà passé dans le langage courant, mais avec un autre sens, hejdeggérien cette fois. L'ajout des termes allemands dans le texte, ou en note, constitue une solution moins élégante, et à laquelle il ne faut certes pas recourir trop souvent, mais qui est largement utilisée : dans la traduction d'œuvres de philosophie grecque, par exemple. Elle rappelle au lecteur qu'une traduction, aussi parfaite soitelle, ne peut remplacer le texte originel.

Car c'est à une rude école de rigueur philosophique que nous invitent G. Jarczyk et P.-J. Labarrière, comme par antidote - souvent fort salutaire - à la séduction trop facile d'un Kojève. Palliant cette austérité, se trouvent à chaque page de la traduction de nombreuses notes explicatives : sur le sens d'un terme, sur la structure du texte - et sur sa signification philosophique. Souvent, en effet, les traducteurs ont ajouté des notes qu'ils dénomment eux-mêmes spéculatives; et le lecteur leur sera reconnaissant d'avoir parfois failli à leur propre volonté de concision, et d'avoir cédé à la tentation de donner plus d'explications. Car celles-ci sont non seulement judicieusement placées et fort éclairantes, mais aussi, la plupart du temps, incontestables. C'est toute une interprétation de la Phénoménologie de l'esprit, fort différente de celle à laquelle nous étions accoutumés, mais fondée sur une connaissance maîtrisée de tout le corpus hégélien, qui se dessine en bas ou au fil des pages : G. Jarczyk et P.-J. Labarrière nous montrent qu'il y a dans la Phénoménologie de l'esprit, comme dans l'Encyclopédie des sciences philosophiques, une structure, une logique. De fait, la « logique » de la Science de la logique est déjà là dans ses grandes lignes, même si ce n'est que « derrière la conscience » - si bien qu'il est tout à fait possible de parler d'une véritable «coextensivité spéculative entre les deux œuvres " (p. 30). Et il n'est rien d'étonnant à cela : la Phénoménologie de l'esprit constitue la synthèse magnifique, le résultat de toute la méditation de Hegel pendant les années de son séjour à Iéna. Pendant ces années (de 1801 à 1806), Hegel n'avait, certes, presque rien publié. Dans de multiples conférences et manuscrits dont aucun, il faut le dire, ne fut publié de son vivant - et certains ne le sont pas encore - il avait pourtant déjà posé les bases de son système futur : de sa Logique, de sa Philosophie de la nature et de sa Philosophie de l'esprit. Certaines des distinctions qu'il établira plus tard, dans l'Encyclopédie, par exemple, entre l'« esprit subjectif » et l'« esprit objectif », ou encore entre l'«esprit objectif» et «l'esprit absolu», n'apparaissent pas encore; et les termes qu'il emploie pour les désigner sont quelque peu différents. Mais la «philosophie de l'esprit ", au sens où le terme est utilisé dans l'Encyclopédie, est bien déjà là; et c'est parce que Hegel, en 1806, l'a déjà élaborée, qu'il peut rédiger la Phénoménologie de l'esprit. 
Mais qu'est-ce donc que cette " philosophie de l'esprit », présentée ici de manière éblouissante? Elle s'apparente, notent discrètement les éditeurs, « aux considérations engagées par les plus grands mystiques d'Occident, un maître Eckhart, en particulier... » (p. 45). Cette remarque, qui mérite d'être développée en d'autres lieux, ne manquera pas, on s'en doute, de susciter un nouveau débat sur la Phénoménologie de l'esprit, prouvant ainsi l'actualité toujours présente de ce chef d'œuvre.

Myriam Bienenstock

Heinrich HeINE, Sur l'histoire de la religion et de la philosophie en Allemagne. Prés., trad. et notes de Jean-Pierre Lefebvre. Paris, Imprimerie nationale, 1994. $15 \times 20,5,226$ p. ( La Salamandre »).

Gerhard HöHN, Heinrich Heine, un intellectuel moderne. Paris, Presses universitaires de France, 1994. 13,5 × 20, 192 p., index (« Perspectives critiques »).

Édouard Gans, Chroniques françaises. Un hégélien juif à Paris (1825, 1830, 1835). Textes prés. et éd. par Norbert W ASZEK, trad. de l'allemand par Myriam BiENENSTOCK. Paris, Cerf, 1993. 13,5 × 21,5, 260 p., index, bibliogr. (« Bibliothèque franco-allemande $»$ ).

La parution simultanée d'une nouvelle traduction de L'Histoire de la religion et de la philosophie en Allemagne, due à Jean-Pierre Lefebvre, et de l'essai de Gerhard Höhn devrait fournir l'occasion de réviser le cas de Heine et de lui rendre la place éminente qui est la sienne dans le débat sur la création littéraire et l'engagement de l'écrivain, celle d'un « intellectuel moderne» avant la lettre, avant Zola et Sartre autour desquels s'est cristallisée la problématique de l'« engagement ".

De fait, la personnalité du poète et penseur Heine n'est pas réductible à l'image du " romantique défroqué », celui des souffrances de l'amour immortalisées par les lieder de Schumann, celui de l'inspiration, en quelque sorte folklorique, de la "Lorelei » et des « Deux Grenadiers ", longtemps populaire en France, aujourd'hui largement oubliée. En revanche, Heine le hégélien de gauche, le journaliste déchaînant les foudres de la censure, le penseur et poète de la modernité, témoin engagé des révolutions libérales et sociales de son temps, n'a rien perdu de son actualité et peut figurer au panthéon des représentants de l'esprit européen héritier des Lumières, pacifiste et cosmopolite.

Choisissant de mettre l'accent sur cette dimension de la personnalité de Heine, encore mal perçue par le grand public français, et d'accorder dans cette perspective une place centrale aux années parisiennes de Heine, l'auteur retrace tout d'abord la "genèse de l'intellectuel ", dans le contexte de l'Allemagne de la Restauration. La censure, le philistinisme triomphant, la trahison des clercs conduiront finalement Heine à choisir l'exil parisien, en raison de ses affinités électives avec l'esprit français. C'est ainsi que Harry Heine devient Henri Heine, le médiateur exemplaire entre les deux cultures.

La seconde partie de l'essai, consacrée aux différentes formes que prend chez Heine le «métier » de l'intellectuel, analyse successivement l'activité du journa- 
liste, du penseur, du critique et du poète engagé des derniers recueils poétiques. À la modernité des sujets correspond la créativité de l'écrivain inventant les formes adaptées à l'appréhension de réalités nouvelles. Le journaliste se signale par l'efficacité de ses chroniques (qui fraient la voie au grand journalisme politique du $\mathrm{xx}^{\mathrm{e}}$ siècle, pensons au reportage sous la République de Weimar), bravant la censure par une stratégie de travestissement ironique propre à faire passer les idées en contrebande, à faire aussi, en son temps, détester le style de Heine. Le penseur, profondément marqué par Hegel, dont il a suivi les cours à Berlin, se sert du modèle de la philosophie de l'Histoire pour rédiger sa brillante Histoire de la religion et de la philosophie en Allemagne (1834). Rompant avec la vision d'une Allemagne contemplative et passive, celle de Madame de Staël, Heine impose une interprétation révolutionnaire de l'histoire de la philosophie allemande, conçue comme pendant théorique et correspondant de la Révolution française. L'ascension progressive vers la conscience de la liberté commence avec Luther, fondateur de la liberté de pensée (en ce sens précurseur de l'Aufklärung, et non représentant d'une régression dans le Moyen Âge), se poursuit avec la révolution philosophique, de Descartes et Spinoza aux Lumières et à l'idéalisme allemand qui en est le terme, pour déboucher, dans une apocalypse future, sur une véritable révolution allemande, la pensée précédant l'action " comme l'éclair le tonnerre ». Cet essai impose Heine comme le premier représentant des Jeunes Hégéliens. C'est en effet «le premier texte qui envisage un usage subversif, et même révolutionnaire de la philosophie hégélienne ", comme le souligne avec force J.-P. Lefebvre, rappelant dans sa préface l'importance de cet ouvrage dans la diffusion, à l'échelle mondiale, de l'image d'une autre Allemagne, celle de l'intelligence progressiste dont les Nazis brûlent les œuvres dès 1933.

Mais la pensée de Heine n'est pas tout entière sous le signe de l'optimisme progressiste (qu'il s'agisse de l'héritage hégélien ou de celui du socialisme utopique, on connaît en effet l'influence des thèses du saint-simonisme sur Heine dans les années 1830). Heine est aussi celui qui a vécu et réfracté dans son œuvre la problématique spécifique de la modernité, le déchirement; à l'époque où d'autres écrivains sont tentés par le repli sur la subjectivité, Heine se confronte à la réalité d'un univers désenchanté, aliéné, qui requiert désormais moins la rêverie du poète que la lucidité intransigeante de l'intellectuel pour le déchiffrer. C'est le sens des attaques féroces par lesquelles Heine rejette les représentants de l'École romantique dans le camp de la réaction. L'intellectuel a ici la vertu de mobiliser les forces, c'est lui qui trace la ligne de front, clarifie les enjeux pour la conscience collective dans l'espace public.

Le problème que pose cette cuvre, et c'est l'horizon ouvert par l'auteur de cet essai, tient dans la difficulté de penser l'unité profonde de la démarche créatrice en résistant à la tentation de disjoindre le poète lyrique et le penseur engagé. À ce propos, on notera que, contrairement aux représentants de la Tendenzliteratur de son temps, Heine a maintenu la dimension de liberté et de gratuité de la création artistique, tout en l'ouvrant aux grands problèmes de son temps et aux contradictions intrinsèques de la modernité. Les analyses consacrées à la subversion des postulats esthétiques du classicisme par l'ironie, la critique, la dissonance, débouchent sur la question plus vaste de l'esthétique du Zeitgedicht - et c'est une question que l'on pourrait soulever à propos de toute littérature d'actualité. L'écrivain engagé est des- 
tiné à assumer une fonction d'éclaireur, à s'exposer aux avant-postes des combats pour l'évolution de l'esprit de l'époque. Ce qui implique réaménagement, mais non abandon d'une dimension proprement artistique. La littérature, en ce sens, devient action dans et sur une époque elle-même en mouvement, dont il s'agit de saisir l'esprit pour l'éclairer et le faire avancer. Les jugements sévères de Kraus et d'Adorno, ce dernier sauvant le prosateur pour condamner le poète des débuts, souligneraient, s'il en était besoin, la difficulté, aujourd'hui comme hier, d'articuler les deux dimensions de l'art et de l'engagement sans en réduire aucune.

La publication de textes d'Édouard Gans (1797-1839), regroupés sous le titre de Chroniques françaises, vient tirer de l'oubli une figure marquante de l'histoire des idées : disciple de Hegel dont il publia, entre autres, les Principes de la philosophie du droit; historien du droit universel, connu pour son magistral ouvrage sur le droit de succession, adversaire de Savigny dans la fameuse querelle entre école historique et non historique; familier du salon berlinois de Rahel Varnhagen et ami de Heine, qu'il a largement initié à la philosophie de Hegel; membre fondateur de l'Association pour la culture et la science du judaïsme, qui jette les fondements d'une science moderne du judaïsme.

On ne manquera pas de souligner la parenté de sa situation avec celle de Heine. Comme lui, il fait partie de cette catégorie d'intellectuels juifs allemands, tournés vers la France et qui, à ce titre, jouent un rôle de premier plan dans les échanges d'idées entre la France et l'Allemagne aux $\mathrm{xIX}^{\mathrm{e}}$ et $\mathrm{xx}^{\mathrm{e}}$ siècles; comme lui, il paie le billet d'entrée dans la culture européenne en se convertissant au protestantisme. Le judaïsme est la donnée première de sa condition : c'est parce qu'il n'arrive pas à obtenir un poste de professeur de droit, à la suite d'une interprétation restrictive du décret sur les Juifs, que É. Gans, en 1825, part pour la France. L'impulsion est alors donnée à une carrière dont un moment important constitue la médiation entre l'Allemagne et la France. Diffuseur de la pensée de Hegel en France (auprès de Victor Cousin notamment), É. Gans, à son retour, joue le même rôle de médiateur des réalités françaises en Allemagne.

C'est cet aspect de l'œuvre et de l'influence de É. Gans que l'éditeur du texte, le spécialiste N. Waszek, a choisi de retenir ici, en analysant cette figure de l'histoire des idées comme illustration de la notion féconde de transferts culturels, dans une préface remarquable, tant par la profondeur de l'érudition que par la clarté et la maîtrise de la présentation. Accessibles pour la première fois en français, les textes réunis ici, extraits des mémoires de É. Gans, concernent ses trois longs séjours en France, de 1825, 1830, 1835. On sera frappé d'emblée par la densité peu commune de ces pages, qui constituent un document d'une extraordinaire vivacité sur la France de l'époque charnière entre la Restauration et la monarchie de Juillet. En effet, ce n'est pas en spectateur passif, mais en observateur averti et parfaitement informé que É. Gans brosse le tableau de la société française, guidé en cela par les principes de la philosophie hégélienne de l'histoire, qui lui fournit un instrument d'analyse efficace et lui fait embrasser naturellement les grandes perspectives historiques (c'est lui, par exemple, qui prophétise à son ami Saint-Marc Girardin la révolution de 1830 et l'arrivée au pouvoir du duc d'Orléans!). Par le biais de l'évocation des figures marquantes du monde politique et scientifique qu'il fréquente assidûment, É. Gans illustre les grandes tendances de l'évolution qui se dessine, saisit les 
tensions, discerne les lignes de forces futures. Qu'il s'agisse des portraits en regard des deux monarques de la Restauration, de l'enthousiasme de la foule portée par les espoirs de la révolution libérale, suivi de la morosité politique de la monarchie de Juillet, de l'évocation de la nouvelle génération d'historiens - Thiers, Mignet, Michelet - des grandes figures qui animent les débats parlementaires, du journal Le Globe - porte-parole de la révolution romantique -, de l'analyse pondérée du saint-simonisme, du salon de Madame Récamier, bref, l'ensemble de ces pages restitue une vision ample et cohérente de l'espace public français. Les possibilités de comparaison qu'il peut établir avec les réalités allemandes de l'époque donnent un accent particulier à ce tableau d'une France admirée par les milieux progressistes allemands, qui la voient mener les grands combats pour la liberté de la presse à une époque où l'évolution de l'Allemagne est durablement entravée par les forces de la réaction. Il est clair, à lire ces pages, que l'identité nationale allemande libérale se forme et s'éduque au contact de la réalité politique française, même si, à cette époque, les Allemands peuvent se réclamer d'une identité culturelle. En témoigne le discours de 1825 prononcé à Paris, où l'hommage rendu à Gœthe prend les accents d'un manifeste d'union nationale : « Nous avons une langue en commun. Celle-ci a un prince et une loi. Ce prince est Gœthe et les œuvres de Gœthe en sont la loi. »

Ces trois ouvrages, à des titres divers, et avec l'éclairage qui leur est propre, devraient contribuer à donner corps à une image de l'Allemagne longtemps méconnue mais qui, avec les travaux récents sur la génération du Vormärz par exemple, s'impose progressivement à l'attention du public français : une Allemagne engagée et progressiste, en dialogue d'idées avec la France, dont les intellectuels apportent une contribution majeure à l'édification de l'idée européenne.

Anne LaGNy

Anna Maria TRIPODI, Fondamenti della gnoseologia critica contemporanea. L'Aquila-Rome, Japadre Éd., 1992. 14 × 20,5, 125 p. ("Categorie Europee", 32).

Le titre de ce livre peut paraître ambigu. « Gnoséologie critique » : il s'agit d'une analyse des problématiques soulevées par la philosophie d'Antonio Rosmini (17971855 ), chef de file du « spiritualisme (ou ontologisme) critique » italien au XIx ${ }^{\mathrm{e}}$ siècle. Pourquoi des «fondements "? A.-M. Tripodi décrit le processus historique de révision du cogito cartésien, qui prend son essor, en Italie, au début du siècle dernier, et vise à reconduire la gnoséologie rationaliste à sa propre fondation métaphysique, au sens d'une fondation de l'acte du connaître en tant que transcendantale et critique, sans pourtant déboucher sur un modèle de subjectivisme de type kantien.

Descartes commence sa «réflexion » à partir de la position du sujet pensant; Locke, par contre, revient à la simple « sensation "; et le philosophe italien veut prendre en compte "l'analyse de la cognition simple qui, s'interposant entre les 
deux, en constitue la clé de lecture; et précisément par cette opération Rosmini rapporte la gnoséologie à son fondement métaphysique » (p. 25).

L'« erreur» de Descartes consisterait dans le remplacement subreptice de l'évidence immédiate du Cogito par un acte de " réflexion " non immédiat (une " cognition complexe $\gg$ ) qui relève d'un rapport plus originaire entre le doute et l'exister. L'erreur consiste dans l'ergo posé entre le cogito et le sum. Ainsi Descartes fait précéder logiquement l'observation par la « démonstration", ce qui le conduit à s'engager dans la recherche d'un critère de certitude qui laisse de côté le problème de l'origine des cognitions. «Le premier but de la philosophie n'est pas la certitude des cognitions, mais plutôt les cognitions elles-mêmes, leur existence, leur origine, de laquelle découle, comme un corollaire, le principe de la certitude. À ce propos Rosmini utilise l'expression d' ignorance méthodique". »

A. M. Tripodi avance une observation importante : «Descartes aurait pourtant refusé de prendre au sérieux l'objection selon laquelle son cogito ergo sum demandait au préalable, comme primum, l'idée d'existence, le principe de toute la philosophie; par contre il aurait objecté qu'il était question d'une intuition immédiate, antérieure à tout raisonnement, sans s'apercevoir que le donc (ergo) contredit sa réponse : c'est-à-dire qu'il ne reconnaît pas la présence d'un raisonnement précédant le cogito, qui révèle un rapport entre l'acte de douter et l'exister. Pour l'instauration d'un tel rapport, pour formuler un tel jugement, il faut connaître l'"exister" comme indépendant à l'égard du "juger" » (p. 26).

Descartes se trompe sur le plan de la capacité analytique du cogito. Il ne s'aperçoit pas qu'il faut « décomposer la pensée en "conscience première" et "actes du penser" »; Descartes n'a pas su analyser la « perception intellective » en ignorant la nature authentique de la « réflexion » qui pense. Voici le cercle vicieux : il établit que la perception distincte est un critère de certitude, et par cela même arrive à démontrer l'existence de Dieu. Grâce à cette présence divine, acquise, il s'assure ensuite du fait que nos perceptions distinctes - qui peuvent toujours nous tromper - viennent de Dieu, ce qui nous garantit qu'elles ne nous trompent pas.

Descartes s'est bien rendu compte que la simple perception de soi n'est pas un élément suffisant pour fonder nos connaissances universelles et nécessaires; il faut quelque chose d'autre, pour que cette perception soit « plus autorisée ". Un tel appui de la perception subjective est "l'idée de l'être, qui a en soi essentiellement l'objectivité et la nécessité ». Ne disposant pas de ce principe, Descartes a recours à l'idée de Dieu, en tombant dans le cercle gnoséologique. A. M. Tripodi nous introduit au cœur de la gnoséologie rosminienne (chap. I, «Subjectivité et objectivité »).

L'idée de l'être peut fonder métaphysiquement un critère de certitude de nos connaissances sensibles. Elle est une idée à la fois transcendantale et objective, en tant qu'elle coïncide avec l' «être commun " (essere comune) concret et « subsistant ». Le cogito n'est pas distinct des « actes transitifs de la pensée » qui forment la conception d'une «pensée immanente». Nous retrouvons le lien entre «l'âme rationnelle " et le corps à travers la corrélation entre ces actes, entre res cogitans et res extensa, entre subjectivité active et objectivité produite. Ce lien organique, qui fonde la corrélation des actes de pensée, c'est l'idée de l'être, le Dieu du philosophe.

La philosophie de Rosmini vise à l'identification des principes relatifs à l'activité du sujet pensant, qui permettent l'accomplissement de la construction d'une connaissance « intégrale » et unitaire du monde, métaphysiquement fondée hors des 
cadres du dualisme kantien. Selon A. M. Tripodi, Rosmini dépasse le subjectivisme intellectualiste de Kant sur le plan ontologique par le passage du domaine de l'interrogation gnoséologique au domaine de l'interrogation ontologique. Qu'est-ce qui permet (et fonde) la corrélation continue des données simples de la perception intellective? Qu'y a-t-il de réel pour la conscience si ce n'est cette idée fondamentale de ce qui est l'être, unité absolue antérieure, la plus vraie et la plus objective car « commune " à la série des aperceptions?

Pour cette tâche méthodique - la recherche des principes du connaître - il y a nécessité d'un «philosophe de l'être », non pas d'un «philosophe du connaître ". Le premier prend en compte l'antériorité de "l'uni-totalité » de l'être, il absorbe l'instance gnoséologique dans le cadre du problème ontologique. Ce n'est pas un hasard si, en Italie, Rosmini a été récemment redécouvert avec intérêt par l'ontologie et les phénoménologues comme un philosophe qui, le premier, a indiqué le chemin pour sortir du " "pré-jugé" rationaliste, qui impose à l'homme de ne rien tenir pour vrai, hors de ce que sa "raison naturelle" lui témoigne comme vrai » (p. 35). L'objectivité du savoir est la conscience de la finitude humaine et, en même temps, «potentialité infinie » à l'intérieur de ces limites.

Rosmini se réclame de Galilée, dans sa découverte d'une « objectivité retrouvée » qui est synonyme d' $d$ indépendance des choses par rapport au sujet », en s'engageant dans une fondation métaphysique de cette objectivité. A. M. Tripodi observe que «l'unité et la positivité essentielle du logos sont des conditions qui fondent le discours philosophique. Il n'est pas permis, à l'homme, d'accéder à un "absolu connaître" qui est le propre de Dieu; cependant il lui est permis d'accéder à un "connaître absolu" par rapport à la forme - à l'idéal. L'intuition de "l'idée de l'être" est le fondement de la pensée rosminienne. Elle est porteuse de l'objectivité, est en mesure de faire concevoir l'ens à l'esprit humain (mente), tel qu'il est en soi et pour soi, dans sa vérité, indépendamment des conditions subjectives particulières de l'esprit qui le pense ".

L'interlocuteur de Rosmini est évidemment Kant. Après avoir analysé, au chapitre II, la «méthodologie de la synthèse » - le lieu où l'« organisme philosophique » de l'ontologie se produit, fondant l'anthropologie et la psychologie, dont la particularité est celle de se poser en tant que «encyclopédie » et «système de la vérité du savoir »-A. M. Tripodi aborde le problème de « Rosmini et la gnoséologie du criticisme ». « Rosmini se pose dans la lignée d'un "idéalisme objectif", pour lequel l'idée est objet de l'esprit et non pas un contenu subjectif de la conscience, ni une simple forme du connaître; la vérité est donc pour lui objective et transcendante, et la pensée n'est possible que comme une pensée de la vérité, indépendante d'elle, "

Kant se serait borné à l'analyse de la « perception», sur le plan d'une recherche des conditions intellectuelles. Il a distingué la «matière " (sensation) par rapport à sa « forme » (le principe de nécessité et d'universalité), un a priori et un a posteriori, en s'interdisant l'accès aux processus qui conditionnent non pas la perception mais la cognition et l'abstraction. Kant dresse une table des catégories - «notions universelles qui entrent dans la formation d'un ens corporel tel qu'il est conçu » mais il n'a pas réfuté l'idéalisme de Berkeley; il transpose l'idéalisme du plan des sensations à celui de l'entendement des sensations. La critique de Rosmini est pointée sur l'idéalisme transcendantal, incapable de «garantir quelque chose de réel en 
soi, hors de la portée humaine », et il débouche directement sur une forme plus subtile et désespérée de scepticisme.

L' « erreur fondamentale du criticisme " consisterait en ceci : " Avoir transformé en subjectivations les objets de la pensée. » Il en résulte que Kant «n'a pas bien approfondi la nature de cet acte intellectuel par lequel nous percevons les choses ".

A. M. Tripodi analyse les résonances de la problématique dans le cadre du débat culturel italien : aujourd'hui Rosmini y demeure un point de repère important mais l'auteur ne manque pas, ensuite, de rappeler son envergure européenne. Le congrès international de Rome, sur « Rosmini penseur européen », en 1988, marqua le début d'un approfondissement de la pensée rosminienne par rapport aux grands courants de la philosophie française et allemande.

Paolo Quintili

Les Courants antimaçonniques hier et aujourd'hui. Éd. par Alain DiERKENS. Bruxelles, Éd. de l'université de Bruxelles, 1993. $16 \times 24,175$ p. (« Problèmes d'histoire des religions », 4).

On ne saurait trop féliciter l'université libre de Bruxelles d'avoir consacré son colloque annuel de 1993 aux courants antimaçonniques : un sujet que l'on flaire souvent mais que l'on a du mal à appréhender. Disons tout de suite que le pluriel s'imposait. Pour plusieurs raisons. Les responsables ont voulu balayer large : dans le temps, ce qui nous vaut un étalement qui va de 1738 à 1992; non moins dans l'espace, puisque le regard s'étend, au-delà de l'Occident, jusqu'en Amérique latine, d'une part, vers les Balkans, la Turquie et même l'Iran, d'autre part. Et ne parlons pas de la variété des approches : ici et là autour d'un personnage, ailleurs plus globale, parfois plus schématique. En guise de conclusion, Hervé Hasquin présente quelques réflexions (trop?) brèves mais d'une grande force. À défaut de pouvoir suivre les divers chapitres qui souvent représentent déjà un condensé de recherches "pointues", nous en resterons à un découpage, explicite ou implicite dans l'ouvrage, qui distingue trois types d'antimaçonnisme : le religieux, le politique et le populaire, sans pourtant nous voiler leurs imbrications.

Pour le premier, on songe tout de suite à la condamnation romaine de 1738 qui a joué un rôle capital. Il importe toutefois d'en bien voir les prolongements plus efficaces sous les pontificats de Pie X et surtout de Léon XIII, avec, en particulier, l'encyclique Humanum genus en 1884 et le congrès de Trente en 1896. Mais l'antimaçonnisme se retrouve dans d'autres confessions chrétiennes, même dans d'autres religions. On le trouve ainsi dans les Églises orthodoxes de Grèce et de Roumanie, voire dans l'une ou l'autre confession de Grande-Bretagne. On ne s'étonne pas qu'en Iran la théocratie shi'ite ait persécuté la maçonnerie. Mais il est plus curieux qu'en Turquie, dans les années 1871-1872, trois ouvrages différents traitent les francs-maçons comme une organisation visant à christianiser les musulmans. Même s'il existe des explications ponctuelles, le fait ne manque pas de piquant.

On s'étonne moins des ressorts politiques de l'antimaçonnisme. Si tout pouvoir se méfie des sociétés secrètes, les régimes dictatoriaux ne pouvaient que combattre une 
institution empreinte d'humanisme libéral. C'est vrai - pour ne pas remonter plus loin dans le temps - du communisme, du nazisme, du franquisme, de Vichy. Diverses communications montrent même qu'une opposition plus discrète reposait sur une anglophobie liée au rôle de la Grande-Bretagne dans la naissance de la maçonnerie. Et une méfiance analogue va s'étendre à des organismes comme la SDN, l'ONU, voire Amnesty international. On peut vraiment parler du polymorphisme des diverses formes de procès ou soupçons antimaçonniques.

L'aspect qui semble le plus neuf dans ce colloque est l'éclairage, par touches diverses, sur l'antimaçonnisme populaire. Celui-ci connaît des formes qu'on dirait presque bénignes: ainsi lorsqu'on traite les frères d'ivrognes rassemblés pour des beuveries, voire d'homosexuels. Bien plus redoutable est l'agressivité qui, surtout à partir de la fin du $\mathrm{XIX}^{\mathrm{e}}$ siècle, associe maçonnerie à satanisme, meurtres rituels, etc. Cette diabolisation, pas tout à fait nouvelle, tient beaucoup à la conjonction de deux phénomènes : les «révélations" invraisemblables faites par Léon Taxil, ancien maçon (ou se disant tel) et la publication d'un faux antisémite, le Protocole des sages de Sion, dévoilant les soi-disant complots politiques des Juifs. Assimiler les deux était vraiment trop tentant pour des populations toujours portées à trouver à leurs malheurs des explications simplistes. Plutôt que d'en évoquer des manifestations multiples, nous faisons nôtre un passage de Jacques Marx dans l'avant-propos de l'ouvrage: après avoir diagnostiqué que «l'antimaçonnisme pousse sur les débris de l'inconscient collectif », il le caractérise comme "une réaction affective, d'allure névrotique; ce qui rendrait d'ailleurs bien utile une psychanalyse de la critique maçonnique ». Si l'on doit souhaiter que se développent les rencontres (évoquées ici ou là) entre responsables de la maçonnerie et d'organismes traditionnellement hostiles, il est à craindre que presque tout restera à faire sans cette opération de salubrité publique.

Louis PÉrouas

La Philosophie allemande de Kant à Heidegger. Sous la dir. de Dominique FolSCHEID. Paris, Presses universitaires de France, 1993. $15 \times 21,5,436$ p., index ( Premier cycle $»)$.

Dans sa présentation, D. Folscheid précise que le classement des auteurs étudiés est, en son fond, «d'ordre chronologique, pour suivre la pente naturelle des esprits en matière d'histoire de la philosophie, donc faciliter l'accès " (p. 5).

Les analyses présentées sont particulièrement bien structurées, approfondies, documentées : l'accès aux auteurs étudiés tient compte des travaux les plus récents, tout en répondant à l'objectif central de ce beau volume qui est de s'en tenir toujours à l'essentiel. Philippe Choulet, André Clair, Françoise Dastur, Dominique Folscheid, Francis Kaplan, Jean-François Marquet, Frédéric Nef, Édouard Sans, Miklos Veto ont participé à ce travail collectif.

Outre les auteurs philosophiques qui ont marqué de façon décisive l'époque qui va « de Kant à Heidegger », sont étudiés des auteurs « d'importance moindre (à des 
titres multiples) » qui ont été « répartis en deux groupes. Les uns, qui gravitent autour d'un auteur majeur, ont été placés à la fin de certains chapitres dans une rubrique intitulée "Autour de...". Ceci devrait permettre de suggérer des connexions et des éléments de discussion, en écho aux débats du temps. Les autres, qui peuvent être rattachés à une école (par exemple, l'école dite "de Francfort ") ou à une préoccupation dominante (par exemple, l'éthique ou la politique), ont été groupés dans des chapitres adéquats » (p. 5).

L'originalité de ce volume tient sans doute dans l'organisation en deux grandes parties des études qui le composent : dix chapitres concernent les auteurs les plus importants (de Kant à Heidegger), sept chapitres traitent de thèmes essentiels pour la compréhension de ces auteurs : ainsi les « sources austro-allemandes de la philosophie analytique " ou les «philosophies de la culture " (de Dilthey à Spengler), «Éthique et existence » (Scheler, Jaspers, Jonas) ou «Littérature et philosophie » (de Lessing à Broch et Jünger).

Les informations bibliographiques sont présentées de façon raisonnée, avec des conseils de lecture.

Une Chronologie politique et culturelle (p. 403-417), une Bibliographie générale (p. 419-420), un Index des termes allemands cités (p. 421-423), un Index des notions (p. 425-431) et un Index des noms propres (p. 433-434) terminent le volume.

Ce livre, dont la présentation d'ensemble est très soignée, constituera un instrument précieux pour développer chez les étudiants en philosophie l'habitude d'une réflexion exigeante, informée et affrontée aux interrogations qui appartiennent à l'horizon ultime de signification de la pensée et des systèmes philosophiques étudiés.

Marie-Jeanne KöNIGSON-Montain

« Husserl », Kairos, 5, 1994.

Ce dernier numéro de la Revue de la faculté de Philosophie de l'université de Toulouse s'ouvre sur un article où $\mathrm{R}$. Bernet rappelle, sur le cas exemplaire de la perception, la prégnance des motifs téléologiques dans la phénoménologie. La notion d'esquisse n'a de sens qu'en relation à une chose en soi visée dans l'anticipation sans jamais être véritablement atteinte. C'est donc dès le début que l'esprit est guidé par cet idéal de connaissance dont on sait le rôle qu'il jouera dans la phénoménologie. Deux articles sont consacrés à la Krisis (P. Dupond, «L'idée de vie dans la Krisis » et M. Hébrard, «L'énigme du monde »). Sans le prendre nécessairement pour fil directeur, les cinq contributions restantes traitent, à un degré ou à un autre, de l'entrelacs des motifs logiques et ontologiques dans l'œuvre de Husserl, et sont ainsi conduits à mettre en lumière l'importance de la période préphénoménologique.

F. Dastur évoque ainsi la figure de Lotze, auteur d'une logique en son temps presque aussi célèbre que celle de Stuart Mill, et rappelle que son interprétation 
" géniale » de la théorie des Idées a aidé Husserl à franchir une étape décisive dans la genèse de la phénoménologie, en le libérant du réalisme extrême auquel il avait adhéré un temps sous l'influence de Bolzano. L'article de D. Parrochia examine une autre source de la pensée husserlienne : la théorie des multiplicités, créée par les géomètres Riemann et Grassmann, et qui a servi de modèle à cette théorie des formes de théorie qui, dans le dernier chapitre des Prolégomènes, est donnée comme l'ultime tâche assignée à la nouvelle logique. Contre J. Vuillemin, ou MerleauPonty, qui reprochaient à Husserl de n'avoir jamais adhéré au formalisme qu'en apparence, l'auteur montre la permanence du paradigme mathématique jusque dans les œuvres de 1935. Un troisième article trouve encore son origine dans une question qui remonte à la période préphénoménologique. Depuis trente ans, en effet, D. Føllesdal propose de clarifier les notions phénoménologiques de sens et de noème en les pensant dans leur relation au Sinn frégéen. D. Fisette entreprend d'arbitrer le débat qu'a suscité cette « phénoménologie de la côte ouest »- entendez: des États-Unis — en répondant aux objections qui lui ont été adressées.

J.-M. Vaysse et É. Rigal tentent pour leur part de ressaisir dans son unité l'itinéraire philosophique de Husserl et sont ainsi amenés à s'interroger sur sa cohérence. Si l'on accepte d'associer d'un côté phénoménologie et psychologie, par le biais de l'égologie par exemple, et logique et ontologie de l'autre, il est permis de considérer leur contribution respective comme deux approches jusqu'à un certain point exclusives l'une de l'autre d'une même question. J.-M. Vaysse, dans « Phénoménologie et Ontologie », rappelle tout d'abord comment le retour de la discipline honnie mettait la phénoménologie face à une difficulté, déjà signalée par Cavaillès; il montre alors comment Husserl pensa y échapper en s'engageant dans des recherches généalogiques où la région conscience était elle-même à constituer. " Les recherches psychologiques et logiques de Husserl », de É. Rigal, s'interrogent également sur la légitimité et la fécondité du tournant transcendantal, mais pour porter à son propos un jugement beaucoup plus sévère : après 1907, Husserl aurait assimilé « deux manières de questionner dont il y a pourtant toute raison de penser qu'elles sont profondément irréductibles " (p. 166).

L'ampleur de l'œuvre de Husserl est telle qu'il était nécessaire de faire un choix. En nous invitant à un retour aux sources de la phénoménologie, ce numéro de Kairos corrige ce que pouvait avoir d'unilatérale l'image de Husserl que nous a léguée Merleau-Ponty, et qui reste encore vivace parmi nous.

Michel Bourdeau

Rudolf BERNET, La Vie du sujet. Recherches sur l'interprétation de Husserl dans la phénoménologie. Paris, Presses universitaires de France, 1994. $15 \times 21,7$, 337 p., index ("Épiméthée »).

Bien connu des phénoménologues pour ses responsabilités aux Archives-Husserl de Louvain comme pour ses différents travaux ${ }^{1}, R$. Bernet nous offre un nouvel

1. Nous pensons, en particulier, à cet ouvrage de référence écrit en collaboration avec I. Kern et E. Marbach, E. Husserl, Darstellung seines Denkens, Hamburg, Felix Meiner Ver- 
ouvrage composé pour moitié d'articles parus ici et là, intitulé La Vie du sujet. Le sous-titre explicite clairement son projet : Recherches sur l'interprétation de Husserl dans la phénoménologie. Mais, nous prévient immédiatement l'auteur, plutôt que de polémiquer avec les prophètes de la mort du sujet, il préfère - on le comprend - reprendre et approfondir le débat avec la critique du sujet menée par la phénoménologie elle-même. Ainsi sommes-nous invités à discuter certaines thèses majeures de la phénoménologie dans la perspective husserlienne «d'une subjectivité qui est transcendance dans l'immanence» (p. 2). Trois chapitres forment cet ouvrage, encadrés d'une importante introduction comme d'une non moins riche conclusion.

L'introduction, consacrée à la réduction chez Husserl (et Fink) et Heidegger, permet à R. Bernet de dégager et de confronter la double vie du sujet transcendantal entendu soit comme sujet constituant et spectateur impartial, soit, en termes heideggériens, selon le double mode propre et impropre de l'existence du Dasein. Indiquons immédiatement pour dissiper tout malentendu qu'il ne s'agit pas ici et dans le reste de l'ouvrage - au nom d'un quelconque éclectisme ou syncrétisme phénoménologique - de gommer les différences mais bien plutôt de préciser les positions respectives des uns et des autres.

Le premier chapitre se demande, en prenant appui sur un cours de Heidegger de 1925, Prolegomena zur Geschichte des Zeitbegriffs, dans quelle mesure la transcendance heideggérienne s'approprie la théorie husserlienne de l'intentionnalité. Celle-ci est ensuite, dans un deuxième temps, plus précisément étudiée du point de vue de la notion de noème qui apparaît chez Husserl dans le cours de 1906-1907 et dont la lecture attentive permet à $\mathrm{R}$. Bernet de dégager différents concepts de noème et, ce faisant, d'éclaircir le texte du premier livre des Ideen. Enfin, ce premier chapitre, intitulé "Intentionnalité et intersubjectivité ", s'achève sur l'étude de la notion de monde, saisie dans toute sa diversité, qu'il s'agisse du monde du chez soi (Heimwelt), du monde universel (Allwelt), du monde de la vie (Lebenswelt), du monde environnant (Umwelt), du monde spirituel (geistige Welt), etc. De ces riches analyses, signalons en particulier la critique de la "constitution " husserlienne à propos de laquelle R. Bernet écrit : "Plutôt que de se révéler comme l'œuvre de l'homme, le monde révèle à l'homme la finitude de sa transcendance " (p. 118).

Le deuxième chapitre est entièrement consacré, ce qui ne surprendra guère dans un ouvrage centré sur l'œuvre de Husserl, à la perception. Soulignant, dans un premier temps, l'importance du motif téléologique dans la phénoménologie husserlienne en général, R. Bernet examine sa place dans la phénoménologie de la perception sensible d'un objet spatial. En effet, la perception est inséparable de l'idée au sens kantien - de chose-en-soi - en un sens non kantien. En d'autres termes, elle est guidée par l'idée régulatrice de donation adéquate, véritable telos de la perception; et le caractère irréalisable de ce but conduit l'auteur à élargir sa réflexion sur l'éthique de la volonté de savoir. Dans un deuxième temps, la perception à nouveau donne à $\mathbf{R}$. Bernet l'occasion d'évoquer le différend qui, entre autres à Davos (1929), opposa Cassirer et Heidegger. Précisant en quel sens la perception relève

lag, GmbH, 1989. Quelques chapitres en sont traduits dans le $n^{\circ} 2$, oct. 1994 et le $n^{\circ} 4$, oct. 1996 de la revue de phénoménologie ALTER. 
selon Cassirer d'un comportement symbolique et donc d'une herméneutique, $R$. Bernet montre que cette conception par certains aspects, et en particulier du point de vue de l'opposition du voir (Sehen) et de la vision (Sicht), se situe à mi-chemin entre la doctrine husserlienne de l'intuition catégoriale et l'analyse heideggérienne du comprendre. On le voit, il ne s'agit pas de réduire la discussion à la « lutte entre une philosophie ancrée dans l'Aufklärung et une forme de fondamentalisme militant, mystique et anti-humaniste » (p. 154); et R. Bernet, plus largement, s'interroge sur ce qui sépare l'herméneutique des formes symboliques et l'herméneutique du Dasein. Enfin, ce chapitre sur la perception s'achève sur Merleau-Ponty et une relecture de la Phénoménologie de la perception dans la perspective d'une philosophie de la nature. R. Bernet envisage alors les notions de vie prépersonnelle, de Leib en tant que système symbolique, de connaturalité du corps et de la chose et, manifestant ainsi l'unité de l'œuvre, montre comment la nature dans la Phénoménologie de la perception et l'esprit sauvage dans Le Visible et l'Invisible «sont une seule et même chose » (p. 184).

Du troisième chapitre, il faut sans aucun doute lire le texte, intitulé «Origine du temps et temps originaire », qui met en évidence de profondes analogies entre la compréhension husserlienne et heideggérienne du temps. Dans ce but, l'auteur compare l'unité de l'impression originaire, de la rétention et de la protention d'une part, et l'unité ekstatique horizontale entre présentifier, retenir et être-tendu-vers d'autre part ; de même, le temps vulgaire de Heidegger et le temps objectif de Husserl. $R$. Bernet ne songe pas pour autant à minimiser les profondes divergences, en particulier du point de vue du projet global, qui séparent le maître et le « disciple », s'il est vrai que, chez Husserl, la phénoménologie du temps fait partie d'un programme épistémologique qui vise à valider les sciences de la nature tandis que la fondation heideggérienne du temps objectif par le temps originaire est "en vérité le récit d'une chute (Verfallen), d'une dégénérescence (Degeneration) de l'origine du temps » (p. 212). Signalons seulement, en ce qui concerne le texte suivant intitulé «La présence du passé », que l'auteur y poursuit la confrontation de Husserl et de Heidegger et y met en question la métaphysique sous-jacente aux analyses husserliennes de la conscience du temps en étudiant la présence du passé.

Dans «L'encadrement du souvenir », troisième texte de ce troisième chapitre, l'auteur interroge à nouveau le souvenir, la manière dont le présent appelle le passé et dont le passé s'intègre au présent. Reprenant brièvement les analyses du souvenir développées par Husserl, Proust et Barthes, R. Bernet rappelle entre autres que, pour Husserl, lorsque je me souviens je n'imagine rien, revis tout, c'est-à-dire retrouve le vécu intentionnel dans lequel l'objet passé fut jadis appréhendé. Puis, notre texte souligne le caractère esthétique de la mémoire involontaire proustienne qui jaillit de l'accord entre des sensations appartenant à des temps différents. Enfin, empruntant à Barthes la distinction à propos de la photographie entre studium (ou cadre) qui est du côté du souvenir volontaire de l'intelligence, et punctum, détail qui m'inflige comme une piqûre et relève de la mémoire involontaire, $R$. Bernet s'attache à rapprocher le souvenir volontaire selon Husserl et le souvenir involontaire selon Proust.

Nous recommanderions particulièrement, pour notre part, la lecture du dernier article qui achève ce troisième chapitre et nous propose une lecture critique de $L a$ Voix et le Phénomène. R. Bernet reconnaît d'emblée toute l'importance de ce texte dont les analyses entraînent un débat avec la tradition philosophique tout entière et 
discute, avec une rare clarté, un texte réputé pour sa difficulté. On sera sans doute séduit par la manière dont l'auteur défend Husserl, par exemple, contre la déconstruction derridienne de la réduction qui s'attaquerait à une réduction «plus fantomatique que phénoménologique » (p. 276); ou par la manière dont il reprend l'interprétation par Derrida de la rétention dans son enchevêtrement (Verflechtung) avec l'impression originaire, sans toutefois souscrire à sa compréhension comme figure supplémentaire de l'enchevêtrement de l'expression et de l'indication - dont $\mathrm{R}$. Bernet accorde le caractère phénoménal tout en défendant la validité eidétique de cette distinction. Enfin, si cet article reconnaît la nouveauté de la lecture de Husserl par Derrida, il en dénonce aussi non pas le logocentrisme mais son « linguocentrisme» (p. 295) entendu au sens d'un centrage exclusif de l'interprétation sur le langage.

Dans la conclusion de cet ouvrage, R. Bernet projette de relire Husserl à partir de sa Wirkungsgeschichte et, plus précisément, à partir de la mise en question par la phénoménologie posthusserlienne de l'objectivisme représentationnaliste et du subjectivisme égologique. Ainsi R. Bernet analyse-t-il l'introduction, dans l'œuvre de Husserl, d'une conception egologique de la conscience transcendantale et l'effacement progressif de la différence entre représentations objectivantes et sentiments. Ce travail est alors l'occasion de renvoyer dos à dos J.-P. Sartre et M. Henry pour leur «même fascination - caractéristique de la phénoménologie française tout entière [R. Bernet est un Suisse expatrié] — pour une forme de la subjectivité qui se situerait en deçà ou en dessous du sujet cartésien » (p. 300). Nous ne pouvons naturellement pas exposer dans ce cadre les analyses extrêmement intéressantes qui, par exemple, esquissent plusieurs rapprochements entre Husserl et Freud à propos du moi et de l'affect. Nous nous contenterons de signaler que ces analyses aboutissent à la mise en évidence d'une vie intentionnelle sinon sans sujet du moins sans objet, à l'œuvre dans le rapport du sujet à un monde prédonné comme dans le rapport « impressionnellement-intentionnel " (p. 326) du sujet à lui-même. Sur ce dernier point, R. Bernet se rapproche de $\mathrm{M}$. Henry bien que le caractère impressionnel de l'automanifestation de la vie subjective se réalise, selon lui, encore grâce à une intentionnalité sui generis.

Ni heideggérien ni sartrien ni derridien ni henryien, cet ouvrage toujours attentif à son lecteur - il y a chez R. Bernet comme une rare politesse d'écriture - invite à se frayer son propre chemin en phénoménologie et à y reposer sérieusement la question du sujet.

Philippe CABESTAN

Maurice Blondel, Euvres complètes. T. I : 1893. Les deux thèses. Texte établi et présenté par Claude Troisfontaines. Paris, Presses universitaires de France, 1995. $15 \times 21,5$, XVIII-760 p.

Avec Les Données immédiates de la conscience de Bergson (1889) et La Modalité du jugement de Brunschvicg (1897), L'Action de Maurice Blondel (1893) fait 
partie des grandes thèses françaises de la fin du XIX ${ }^{e}$ siècle. Éditée peu après la soutenance, vite épuisée, la thèse ne fut rééditée qu'en 1950, après la mort de Blondel en 1949. Elle avait suscité un débat philosophique, mais aussi des débats théologiques par lesquels Blondel fut engagé dans cet épisode difficile de l'histoire du catholicisme français contemporain qu'est la « crise moderniste ». Plutôt que de rééditer sa thèse, Blondel en inséra la matière dans sa trilogie comprenant : La Pensée (2 tomes, 1934), L'Être et les êtres (1935) et, enfin, L'Action (tome 1, 1936, tome 2, 1937). Mais, si importante qu'ait été cette reprise au regard de Blondel, elle n'éclipse pas la rédaction première à laquelle il faut toujours revenir pour saisir la nouveauté et l'originalité de la pensée blondélienne. Quant à la thèse latine, elle était encore inédite. Entreprise par Claude Troisfontaines, qui dirige les archives Blondel à Louvain-la-neuve, la publication des CEuvres complètes de Blondel devait donc commencer par les deux thèses soutenues en 1893. Ce qui est fait dans cet ouvrage où l'on trouve des présentations à la fois précises et sobres, et des documents sur la soutenance dont l'intérêt est certain.

L'Action de 1893 porte en sous-titre: Essai d'une critique de la vie et d'une science de la pratique. S'affirme ainsi la volonté de saisir philosophiquement ce que plus tard on appellera l'existence, ou l'engagement du sujet dans le monde naturel et social. S'affirme également le projet de donner à cette analyse existentielle la forme d'une "science de la pratique ". Ce qui implique pour Blondel de saisir la totalité ordonnée des déterminations de l'existence afin de montrer comment la liberté humaine ne peut s'accomplir sans ratifier l'ensemble des conditions qui la rendent possible et sans épouser le mouvement qui la porte vers des fins à travers lesquelles elle poursuit l'adéquation de soi à soi. Une telle méthode est commandée par un présupposé heuristique: la vie humaine est mue par un dynamisme interne qui lui donne une unité profonde et qui constitue également une norme immanente. $\mathrm{Ce}$ dynamisme, c'est la « volonté voulante » que Blondel distingue des projets particuliers et explicites qui tentent de la traduire et de la réaliser, projets constitutifs de la «volonté voulue ». La distinction s'impose lorsqu'on réfléchit sur des orientations contemporaines telles que le pessimisme d'un Schopenhauer ou le dilettantisme d'un Renan et de ses disciples. Blondel montre ainsi que le problème moral se réaffirme au sein même de sa négation et que la volonté affirmée du néant recèle un amour caché et déçu de l'être.

Si la voie du néant est barrée, au moins faut-il affirmer le phénomène : « il y a quelque chose ", ne serait-ce que l'immédiat sensible dont part en effet la partie centrale de la thèse qui parcourt en cinq étapes successives l'ensemble des conditions qui sont de fait ratifiées par le moindre acte de liberté. Plus nous avançons dans cette troisième partie, plus nous voyons se constituer un seul et même organisme comprenant les phénomènes sensibles qu'étudient les sciences positives, la vie intérieure de l'individu, la cité, la société humaine, la solidarité universelle, la république idéale des intelligences, le royaume moral des fins. Au terme de cette partie, « le phénomène universel ne semble exister que pour devenir le thêâtre de la moralité, ou mieux encore pour être le corps même de la volonté » (p. 337).

Un humaniste s'arrêterait là, et c'est bien ce que fait Léon Brunschvicg dans cette Introduction à la vie de l'esprit (1900) dont on n'a peut-être pas assez remarqué qu'elle est une réplique rationaliste à la métaphysique blondélienne du dépassement chrétien. Selon Blondel, la visée du vouloir transcende le Droit, l'Art, la 
Morale, mais aussi la Métaphysique et la Religion. Pour le dire d'un mot, le principe premier et ultime du dynamisme spirituel, c'est l'idée de Dieu, en tant que «nous ne pouvons connaître Dieu sans vouloir le devenir en quelque façon » (p. 388). En définitive, l'homme agissant se trouve confronté à une alternative qui lui impose une option : être dieu sans Dieu et contre Dieu, être Dieu par Dieu et avec Dieu. Dès le départ, l'amour de soi a été repéré comme l'élément moteur de la progression, mais, au terme, il s'agit de savoir s'il s'accomplira dans la préférence absolue de soi ou dans le sacrifice de l'autonomie, permettant l'accueil de l'Autre.

Cet accueil est, pour Blondel, la réponse appropriée à l'offre chrétienne du surnaturel. Ce dernier mot, d'ailleurs peu fréquent dans la thèse, sera la source de bien des controverses. Du côté des philosophes que ce concept théologique inquiète. Et du côté des théologiens qui ont peur de voir "naturaliser le surnaturel ». La suite des CEuvres complètes montrera combien Blondel a été détourné de ses tâches essentielles par des débats assez confus sur la portée apologétique de sa thèse. Cette publication montrera aussi l'importance des articles concernant l'histoire de la philosophie et notamment Spinoza et le spinozisme, objets du grand livre de son ami Victor Delbos. Également d'ailleurs l'attention avec laquelle le philosophe d'Aix suit, malgré la distance, les travaux de la Société française de philosophie.

Il faut relire le document ici présenté sur "Une soutenance de thèse " pour comprendre comment Blondel a peine à convaincre son jury de l'intention proprement philosophique de son travail. La composition de ce jury était d'ailleurs remarquable, puisqu'il allait de Paul Janet, ultime survivant du spiritualisme éclectique, jusqu'à Henri Marion et Gabriel Séailles, en passant par cet excellent spécialiste de la pensée grecque qu'était Victor Brochard, et, bien entendu, le directeur de la thèse, Émile Boutroux, à la fois philosophe des sciences et historien de la philosophie. C'est en fait Brochard qui pose la question cruciale lorsqu'il dit au candidat: «J'aurais voulu pouvoir étudier et vérifier de près l'unité systématique de votre construction. Comme vous prétendez nous conduire fort loin, il est important de regarder si le fil ne se rompt pas en route et si la continuité que vous pensez constater partout n'est pas artificielle » (p. 728).

Malgré la reprise qu'en avait faite Blondel en 1930, la thèse latine De vinculo substantiali et de substantia composita apud Leibnitium n'avait jamais été publiée. Claude Troisfontaines nous donne ici pour la première fois le texte latin et la traduction française de cette thèse, et il y joint un compte rendu de la soutenance préparé en 1893 par Élie Halévy et non publié. Plus encore qu'à la connaissance de Leibniz, cette thèse apporte à la connaissance de Blondel lui-même. Certes, son travail historique est très sérieux, mais l'essentiel aujourd'hui, c'est sans doute ce que nous percevons, à travers le choix et le traitement de ce thème leibnizien, des orientations les plus profondes de la pensée blondélienne. Si l'auteur des thèses de 1893 s'est orienté vers l'action, c'est pour répondre à une question fondamentale : quel est le lien unissant les phénomènes et leur donnant une consistance proprement ontologique? La réponse ultime à cette question sera ce que Blondel lui-même appelle son panchristisme. Mais, en première analyse, le lien des phénomènes, c'est l'homme, et c'est plus précisément l'action humaine. La thèse secondaire et le dernier chapitre 
de la thèse principale dégagent ainsi le sens ontologique de l'analyse blondélienne de l'action.

Pierre CouIn

Lectures philosophiques de Sören Kierkegaard. Kierkegaard chez ses contemporains danois. Textes de J. L. Heiberg, H. L. Martensen, P. M. Möller, F. C. Sibbern, F. Beck et S. A. Kierkegaard. Trad. du danois et prés. par Henri-Bernard Vergote. Paris, Presses universitaires de France, 1993. 13,5 × 21,5, 340 p., index («Philosophie d'aujourd'hui »).

L'ouvrage a pour principal propos de fournir pour la première fois au lecteur français un aperçu aussi synoptique que possible du climat philosophique danois dans lequel évolua le jeune Kierkegaard, tout particulièrement sous l'aspect (alors dominant) des rapports entre christianisme et hégélianisme. On découvrira ainsi la traduction de quatre textes, dont les auteurs comptent parmi les principales figures de la vie intellectuelle de l'époque, ainsi qu'une traduction du compte rendu de la thèse de Kierkegaard Le Concept d'ironie constamment rapporté à Socrate, qui donne en retour un exemple des échos que suscitèrent les premiers travaux de Kierkegaard lui-même auprès de ses contemporains.

Ces textes et, au-delà, les cuvres de leurs auteurs respectifs, sont d'un intérêt philosophique fort inégal : aussi bien ont-ils fort inégalement pesé sur le devenir de la pensée kierkegaardienne.

Ainsi, le lecteur ne trouvera guère, en la personne de J. L. Heiberg ( « De la signification de la philosophie pour l'époque actuelle », 1833) qu'un assez pâle épigone de Hegel dont le principal mérite(?) fut d'être l'introducteur du hégélianisme au Danemark; Kierkegaard, avec raison, n'en fit pas grand cas.

Plus intéressante est la tentative de critique du hégélianisme proposée par H. L. Martensen ( «Compte rendu de la conférence d'introduction au cours de logique [...] de J. L. Heiberg ", 1836); professeur de philosophie dont Kierkegaard suivit un temps les cours, Martensen invite avec certaines intuitions (thèmes de la révélation, de la parole...) à une saine méfiance vis-à-vis de la philosophie hégélienne, sans toutefois parvenir à une radicale rupture avec celle-ci.

C'est avec P. M. Möller que l'on trouvera le plus de consistance philosophique, en même temps que la seule source d'influence véritable du jeune Kierkegaard (rappelons, par exemple, que Le Concept d'angoisse lui sera dédié). Dans le texte de P. M. Möller présenté ici ( $R$ Réflexion sur la possibilité de prouver l'immortalité de l'âme humaine $[. .$.$] », 1837), deux points méritent en particulier d'être mentionnés$ sous ce double rapport : quant à la forme, le procédé consistant à insérer dans le tissu proprement conceptuel du discours une brève mise en scène campant des personnages types et des situations riches de sens; quant au contenu, la farouche affirmation de l'impossibilité pour l'homme de bâtir un quelconque « système de l'existence" (ceia évidemment à l'encontre de Hegel). On mesure suffisamment par là que la dette de Kierkegaard à son égard n'est pas mince. 
Suit un article de F. C. Sibbern ( Remarques et recherches concernant particulièrement la philosophie de Hegel [...] », 1838) qui offre un nouvel exemple d'une tentative d'opposition à Hegel ne parvenant pas à se hisser au niveau d'une véritable réfutation, malgré le louable souci (ordinairement fort négligé) d'entrer quelque peu dans le détail des textes hégéliens visés.

Enfin, le «Compte rendu » (1842) de F. Beck, intelligent et bien écrit, peut être considéré comme une introduction très convenable à la lecture du Concept d'ironie.

Signalons pour finir la traduction d'un court fragment, datant de 1836-1837 ( « Le trouble de l'époque. La confusion des langues »), dans lequel le jeune Kierkegaard raille habilement l'esprit superficiel et verbeux de ses compatriotes contemporains, ainsi que d'intéressantes notes de lecture et de cours s'échelonnant de 1841 à 1844 («Philosophica »).

L'ensemble est précédé d'une substantielle présentation de Henri-Bernard Vergote, laquelle, en dépit de son caractère parfois quelque peu décousu et de sa phraséologie souvent assez alambiquée, abonde en aperçus historiques et philosophiques passionnants.

Gildas RICHARD

Politique et Philosophie dans l'œuvre de Louis Althusser. Sous la dir. de Sylvain Lazarus. Paris, Presses universitaires de France, 1993. $15 \times 21,7,179$ p., bibliogr. («Pratiques théoriques »).

Ce recueil, ni polémique ni apologétique, des neuf exposés du « colloque d'hommage et de discussion » organisé les 29 et 30 mars 1991 par l'université de ParisVIII "autour de l'œuvre" de Louis Althusser, est utilement complété d'une «bibliographie choisie " qui donne l'essentiel; toutefois, puisqu'il s'agit de «politique et philosophie ", mention pourrait y être faite de deux parutions de ce point de vue importantes : dans L'Humanité du 21 mars 1969, un article: "Comment lire "Le Capital"? "; et fin 1969, un "Avertissement » de vingt-trois pages à l'édition grand public Garnier Flammarion du livre I du Capital.

En ouverture, Sylvain Lazarus, instigateur du colloque, intervient sur un fait majeur de l'œuvre d'Althusser : établir la pensabilité du politique (à l'encontre du point aveugle de la philosophie idéaliste), et corrélativement celle des catégories de « parti » et de "classe ». Il s'agit donc de penser la totalité concrète dans ses articulations, de façon rigoureusement philosophique, c'est-à-dire à travers des « énoncés inauguraux [qui] sont des énoncés sur la dialectique de la pensée et de son objet $\gg$.

Les thèses principales sont rappelées par Alain Badiou. « Pour L. Althusser, les questions de la pensée relevaient du combat, de la ligne de front du rapport de force. » En effet, la ligne n'est pas métaphore géométrique visant à assigner rectitude à la pensée; elle est ligne de démarcation permettant de représenter et de conceptualiser la lutte dans le champ des idées. La philosophie est moins un débat (dans la mesure où il n'y a pas de communication philosophique) qu'un combat, 
d'où l'affirmation de l'intervention : il s'agit de délimiter des positions et de déterminer des problématiques pour définir leurs rapports dialectiques et mettre en évidence, à la façon d'Engels, une ligne de démarcation déterminante en dernière instance, à savoir celle qui sépare l'idéalisme du matérialisme. La philosophie n'est ni une théorie de la connaissance («tout le devenir de la pensée d'Althusser sur ce point est celui d'une désépistémologisation de la philosophie ») ni une morale civique : elle ne s'entretient que de son rapport à deux points nodaux extraphilosophiques, la politique et les sciences. Pratique scientifique, mais non science, elle n'a pas d'objet au sens où les sciences en ont un; " les catégories de la philosophie sont vides, car tout leur office est d'opérer à partir et en direction de pratiques déjà données. » La philosophie consiste à produire des thèses (et c'est là sa scientificité) et elle « intervient politiquement ». Reste alors à déterminer ce que recouvre la politique.

Étienne Balibar, pour montrer l'irréductibilité de la philosophie à une simple modalité de la politique, réfléchit la notion d'objet philosophique par l'intermédiaire d'une analyse de la coupure épistémologique - concept qui fonctionne de pair avec celui de «la problématique ». Il en balise la généalogie (qui montre que la production althussérienne s'appuie sur une appropriation critique de l'histoire de la philosophie); puis il discerne cinq moments dans la signification et la portée théorique de ce concept, le dernier étant celui de sa disparition ( « dans ses textes ultimes, Althusser ne mentionne plus la coupure épistémologique $»$ ). Disparue? Ne serait-elle pas plutôt pleinement opératoire de sorte qu'elle n'est plus « visible » comme délimitation de continents ou de problématiques, mais est interne à la pratique philosophique, lui permettant de discriminer l'idéologique au sein même d'une pratique théorique. Si initialement la coupure fut pour Althusser " un objet philosophique », ne devient-elle pas finalement l'instrument de la pratique philosophique? Quant au concept de "topique " sur lequel Balibar insiste, est-il complètement pertinent? Il est statique, et chez Freud précisément, les concepts fondamentaux de la métapsychologie sont ceux de «dynamique » et d' «économique». «Le thème de la "topique" est lui aussi, en dernière analyse, d'origine platonicienne », ajoute-t-il; mais la référence à la «politique de la Caverne » ne suffit pas à légitimer l'affirmation d'un « arrière-plan platonicien » (les deux utilisations du concept de « topique » antérieurement à Freud sont la Rhétorique d'Aristote et la Critique de la raison pure de Kant). Et conclure que «toute l'histoire de la philosophie [...] n'est pas autre chose que l'histoire des renversements du platonisme », n'est-ce pas abolir pour ainsi dire l'autre au bénéfice du même et laisser pointer l'idéologie de l'origine? Le platonisme aurait-il une fois pour toutes épuisé, au moins virtuellement, la pensée et clôt le champ sinon les modalités du discours philosophique? Marx et le marxisme — dont Althusser - répondent sans doute à la question.

Autant les mentions explicites de Machiavel dans les écrits d'Althusser sont rares, autant son enseignement oral en était nourri, remarque d'emblée Emmanuel Terray. Il y voit deux raisons : d'abord, «l'étude des rapports entre "Politique et Philosophie au XviII ${ }^{\mathrm{e}}$ siècle français" ", qui montre la différence entre les théoriciens du contrat et leurs adversaires, en particulier Montesquieu, sur ce point proche de Machiavel; ensuite, «la lecture de Gramsci », afin d'approfondir le matérialisme historique. Or «pour Gramsci, Machiavel est d'abord le théoricien de l'État moderne » qui montre les modalités de son apparition. Puis Terray s'arrête sur la 
conférence de 1978 consacrée à la "Solitude de Machiavel ", dans laquelle Althusser met en parallèle Machiavel et Marx (« ils rendent [...] publics l'usage de la force et le rôle de la violence »). Par son réalisme, Machiavel « apparaît comme un théoricien de la Révolution " qui énonce les conditions de fondation d'une principauté nouvelle. Plus généralement, E. Terray émet l'hypothèse, en s'appuyant sur quelques textes d'Althusser, que «la théorie politique machiavélienne [...] aurait fonctionné comme le substitut d'une théorie marxiste défaillante » de l'État, du Parti et plus généralement de la politique. Enfin, la raison essentielle de l'intérêt du marxisme pour Machiavel serait le caractère matérialiste de sa pensée.

Reste que la lutte des classes rencontre un obstacle de taille : le «sens commun », qui «est quelque chose de trop profondément sédimenté et stratifié pour qu'on puisse se contenter d'agir sur lui par une pédagogie de la communication et de la transmission des idées». Costanzo Preve analyse la tentative d'Althusser «pour modifier philosophiquement [...] le sens commun du militant communiste historique ", en engageant la triple critique de l'historicisme, de l'humanisme et de l'économisme.

Francine Demichel s'attache à "évaluer l'importance d'Althusser dans le développement de la philosophie du droit »; elle établit les caractéristiques de la recherche juridique d'alors face auxquelles il y a un marxisme réductionniste empreint d'économisme, la problématique générale étant la même : celle d' " une conception purement instrumentaliste du dispositif juridique ». « Ce paysage [...] va être modifié en profondeur sous la double pression d'un événement — mai 1968 et d'un chercheur, Althusser » qui « va poser les questions essentielles : pourquoi y a-t-il du droit? Et comment le droit fonctionne-t-il ? »

Reste le thème de «la lecture symptomale chez Althusser " exposé de façon nuancée par J.-M. Vincent; elle consiste « en quelque sorte à produire un autre texte qui éclaire et déplace le premier ». Soumis à une telle lecture, $L \boldsymbol{e}$ Capital n'est plus un texte absolu à validité intangible : ici donc, ni dogmatisme ni immédiateté; d'où les conseils de lecture discontinue du Capital. Notant une «critique de gauche du stalinisme " ni suffisamment globale, ni suffisamment frontale, J.-M. Vincent y voit « un point aveugle sur lequel il y a lieu de s'interroger en soumettant L. Althusser, à son tour, à une lecture symptomale », laquelle permettrait de montrer « la présence discrète » de Heidegger sur laquelle Althusser n'aurait pas réfléchi au motif que la philosophie marxiste trace toujours et encore des lignes de démarcation.

«Pour moi Althusser, c'est la fulgurance de quelques textes et l'éclat d'un échec », avertit Jacques Rancière examinant notamment dans la préface de Lire le Capital "la politique althussérienne de la lecture » qui rompt avec le mythe de la lecture immédiate. Il note «une étrange bévue d'Althusser», en comparant les blancs et "pointillés qui appellent la bonne réponse " au procédé pédagogique qui demande à l'élève de donner les mots manquants dans une phrase incomplète mais déjà connue du maître. En fait, les blancs révélés par la lecture dite symptomale, le jeu du latent et du manifeste, ne sont-ils pas autant d'indices désignant leur lieu d'emprunt : la Traumdeutung? Du coup, il est cohérent que le procès de connaissance soit pensé en termes de production. «Ce qu'il veut penser, c'est la coupure; or ce que la lecture symptomale pense, c'est nécessairement la continuité » puisque "d'une épistémé à l'autre, il n'y a pour Foucault, aucune question ni aucune réponse commune »; ce détour par Foucault est-il pleinement approprié? Le 
concept d'« épistémè » selon Foucault n'a ni la même compréhension ni la même extension que ceux, althussériens, de «continent » ou de «problématique ».

François Regnault, dressant le "Portrait du philosophe », voudrait " communiquer l'enthousiasme d'une découverte qui se résume toute, au fond, en ces années 60, au seul nom de Karl Marx, redevenu grâce à lui un philosophe dans la lignée des plus grands et un savant dans celle de Galilée ». Il insiste sur la hauteur de vue d'Althusser, puis rappelle l'article important, théoriquement et politiquement, sur « Freud et Lacan ». F. Regnault exprime nettement la nouveauté et l'incidence du travail d'Althusser dans la conjoncture philosophique d'alors : c'était « la thèse contre le commentaire, l'axiome contre l'introspection, l'histoire de la philosophie contre la description de la conscience. Et aussi, il faut le dire, Hegel contre Heidegger et Marx contre Sartre. Et puis aussi, bien sûr, le matérialisme contre l'idéalisme et la dialectique contre la métaphysique ", bref la liberté de penser, c'est-à-dire le courage contre l'idéologie dominante et « la faculté de penser des choses extrêmes ». Puis il indique «ce que faute de mieux [il appellera] les aliénations d'Althusser » (en précisant soigneusement le terme) : « d'abord [...], ce qui fut par les lois décrété aliénation mentale " eu égard au meurtre de sa femme; puis « l'aliénation au P.C.F. » qu'il «n'a jamais voulu [...] quitter », parce que finalement le sens du marxisme-léninisme et du communisme ne cessent « d'avoir cours même lorsque les murs se sont effondrés "; enfin « l'aliénation la plus radicale [...], celle de la philosophie à la politique » assez longuement développée à partir de son ressort initial : la dyade justice-force.

Au total, même le lecteur peu averti de la pratique philosophique d'Althusser remarquera que les imputations de scientisme et de dogmatisme portées traditionnellement à son égard apparaissent bien réductrices et intempestives, et cachent des avancées réelles et des débats nécessaires.

Jean-Paul NAMBOT

Domenico Losurdo, Hegel et la Catastrophe allemande. Trad. de l'italien par Charles Alunn. Paris, Albin Michel, 1994, 14,5 × 22,5, 232 p., index (« Bibliothèque du Collège international de philosophie »).

Après Hegel et les Libéraux (Paris, Presses universitaires de France, 1992) et Autocensure et Compromis dans la pensée politique de Kant (Lille, Presses universitaires de Lille, 1993), Hegel et la Catastrophe allemande est donc le troisième ouvrage de Domenico Losurdo, professeur à l'université d'Urbino, auquel sont rendus les honneurs de la traduction. Les deux précédents ouvrages nous avaient déjà convaincus - dans un esprit qui n'était pas sans nous rappeler, à nous autres Français, celui des travaux de Jacques D'Hondt - des ressources, en vue de la compréhension d'une philosophie politique, de sa propre mise en situation politique comme de celle de ses principaux interprètes: si, en général, lorsqu'il s'agit d'auteurs comme Kant ou Hegel, on a toujours une certaine tendance à préférer les explications internes à la Gueroult, il faut bien reconnaître cependant que, lorsqu'il y va de 
l'aspect politique de l'œuvre de ces mêmes auteurs, on ne peut sans dommage se priver des apports d'une lecture externe. Tant il est vrai que c'est lorsqu'elle se fait politique qu'une philosophie se porte à ses propres limites, sur les bords de sa propre marge pour s'y confronter à sa propre extériorité. Cependant, dans les deux premiers ouvrages cités, l'auteur mettait cette approche politique des œuvres politiques de Kant et de Hegel au service d'une explication de ces mêmes œuvres et de leur meilleure compréhension: c'étaient bien les textes mêmes qu'il s'agissait d'éclairer par leur mise en situation politique. Tel n'est pas le cas de Hegel et la Catastrophe allemande: le lecteur qui serait encore ignorant des Principes de la philosophie du droit ne doit pas espérer ici apprendre quoi que ce soit de la pensée même de Hegel et les textes de Hegel ne seront que rarement convoqués. L'enjeu n'est pas ici la pensée de Hegel confrontée aux enjeux et aux luttes politiques de son temps, mais l'image de Hegel après Hegel dans la culture européenne, et ses modifications dans le contexte des guerres franco-allemandes puis mondiales de 1870 à 1945. Autrement dit, on ne cherchera pas ici à lire Hegel en personne, mais on lira ce que d'autres que lui ont lu en lui dans le contexte politique et idéologique qui était le leur, et quel contexte idéologique déterminé a pu faire qu'ils lisent cela, propageant ainsi telle ou telle image de Hegel.

On lira avec grand intérêt les pages (chap. Il : "Les "idées de 1914" ", p. 53-118) consacrées à la formation en Allemagne, dans la seconde moitié du xix ${ }^{e}$ siècle et dans les milieux réactionnaires des nationaux-libéraux (Haym, Treitschke, Troeltsch, Nartop, Sombart, Scheler, Eucken), de l'image-repoussoir d'un Hegel philistin, étatiste, universaliste, égalitariste, jacobin, en un mot, « Français », en tout point contraire à «l'esprit» germanique dont les valeurs cardinales seraient la liberté, l'individualisme (" principe germanique protestant », selon Haym), l'antiégalitarisme et une conception de l'État non pas juridique mais fusionnelle - où le Staat, célébré, divinisé par Hegel, est posé comme nécessairement mécaniste, tandis que lui est opposée l'organicité vivante et libre du Volk. Autant de valeurs que les teutomanes avaient déjà célébrées en leur temps et à la Deutschtum desquelles Hegel en personne avait opposé l'ironie de son Deutschdumm. Mais le plus intéressant dans cette histoire est de voir cette image d'un Hegel étatiste et anti-individualiste, fabriquée de toute pièce par les nationaux-libéraux allemands les plus réactionnaires, être purement et simplement reprise telle quelle par la philosophie de guerre des pays de l'Entente : pour avoir péché en divinisant l'État, voilà le même Hegel transformé, de l'autre côté du Rhin et de la Manche, en penseur le plus représentatif de ce qu'il y a de plus typiquement et dangereusement germanique. Le hégélianisme devient la traduction de ce qu'il y a de fondamentalement « grégaire » (Andler), belliqueux, impérialiste, étatiste, "mécaniste » (Bergson), et anti-individualiste (Boutroux) dans la culture allemande. Cependant, le destin de cette image de Hegel ne s'achève pas ainsi : il était de faire retour en Allemagne à la faveur de la victoire des forces de l'Entente en 1918. Et l'on voit la gauche non communiste et socialdémocrate assumer à son tour l'image d'un Hegel étatiste, impérialiste et prussien, forgée pourtant par les penseurs réactionnaires du siècle précédent. L'étatisme étant entre-temps devenu également synonyme de communisme, c'est contre un Hegel ainsi placé à la source aussi bien de l'étatisme et du militarisme prussiens que de l'étatisme et du collectivisme marxistes-léninistes, que les sociaux-démocrates (notamment Bernstein) préfêrent inaugurer un «retour à Kant» (p. 114-118). Ce 
Hegel posé en père du collectivisme bolchevique ne devait pas seulement être rejeté par les sociaux-démocrates : il le fut aussi par les nationaux-socialistes (Hasse, Baeumler, Rosenzweig) qui opposèrent «le Volk à la morte extériorité du mécanisme étatique » (p. 127). On comprend, dès lors, pourquoi Carl Schmitt a pu dire que le jour de la prise du pouvoir par Hitler fut aussi celui de la mort de Hegel.

On voit tout l'intérêt historique de cette enquête sur l'image de Hegel : son avantage est notamment de dénoncer le caractère idéologique de certaines lectures de Hegel qui, en leur temps, ont pu prétendre à une certaine objectivité philosophique. C'est notamment le cas de la « lecture » de Hegel par Popper (La Société ouverte et ses ennemis, 1945) qui, ainsi remise en perspective, apparaît clairement comme la reconduction de l'image de Hegel issue de la Première Guerre mondiale et de l'idéologie de guerre des pays de l'Entente.

Cependant, d'un point de vue philosophique, cette enquête laisse quelque peu insatisfait : cela tient peut-être au fait que Hegel et la Catastrophe allemande n'est que la conclusion d'un ouvrage plus important, Hegel und das deutsche Erbe. Philosophie und nationale Frage zwischen Revolution und Reaktion (Cologne, PahlRugenstein Verlag, 1989). Il demeure néanmoins que l'objet même de l'enquête (« l'image» d'une pensée) reste vague et non défini, de sorte que l'on hésite constamment quant à la réponse à donner à la question de savoir si l'on a affaire ici à une tentative relevant de l'histoire des idées ou à un essai d'histoire des idéologies. Dans le premier cas, on regrettera l'absence d'une véritable explication avec la pensée politique hégélienne considérée en et pour elle-même : cela aurait permis de savoir s'il y a dans la pensée même de Hegel quelque chose susceptible d'expliquer pourquoi, plus que tout autre, elle peut se refléter en des images d'elle-même si diverses et tellement opposées. Mais si c'est d'une histoire des idéologies qu'il s'agit, alors on est en manque d'une thématisation explicite des rapports entre une philosophie, voire la philosophie, et ses reflets idéologiques : peut-on expliquer qu'une pensée aussi purement rationnelle que celle de Hegel ait pu devenir un enjeu dans les conflits les plus irrationnels et les plus passionnels de la première moitié de notre siècle? Comment une pensée devient-elle l'enjeu de rapports de force concrets, et la simple annexe de Quartiers généraux ennemis? Le terme d'image, si souvent employé par l'auteur, n'explique rien, à moins qu'il ne suppose le problème résolu : en réalité, il ne fait que mal dissimuler un embarras théorique, quand il ne donne pas l'impression d'une concession faite à une certaine idéologisation récente du débat philosophique au moyen de sa médiatisation. La philosophie a déjà fort à faire avec les idées : elle peut laisser les images à d'autres...

Franck FischBACH

Donald DAvidson, Actions et Événements. Trad. de l'américain par Pascal ENGEL. Paris, Presses universitaires de France, 1993. $15 \times 21,5,402$ p., bibliogr., index (« Épiméthée »).

La réputation de Davidson repose sur deux ouvrages parus il y a maintenant dix ans environ. La présente traduction vient donc compléter celle que P. Engel avait 
déjà donnée il y a quelque temps des Enquêtes sur la vérité et l'interprétation. Divisé en trois parties de cinq chapitres chacune, ce recueil d'articles publiés entre 1963 et 1968 apparaît aujourd'hui dominé par deux thèses majeures concernant l'une la philosophie de l'action et l'autre la philosophie de l'esprit. Dans le premier cas, Davidson s'est fait l'avocat d'une théorie causale de l'action, c'est-à-dire d'une théorie qui, à l'encontre de l'enseignement de Wittgenstein, veut réintroduire nos raisons d'agir dans la chaîne causale. Dans le second cas, le philosophe américain a développé un monisme anomal qui accomplit ce tour de force d'être matérialiste sans nier pour autant l'irréductibilité du mental.

Les fragments d'autobiographie intellectuelle contenus dans les derniers essais invitent à voir la théorie causale de l'action comme le résultat d'un triple refus. L'auteur nous apprend en effet qu'il a commencé sa carrière dans le champ de la psychologie expérimentale. En suggérant, en 1926, une procédure qui permette de démêler opinions et désirs, probabilités subjectives et utilités, Ramsey avait apporté à la théorie de la décision, ou théorie du choix rationnel, une contribution dont la valeur ne fut appréciée que bien plus tard. C'est ainsi que, prenant cette théorie comme une hypothèse psychologique empirique sur la façon dont nous nous déterminons à agir, le jeune Davidson s'évertuait à la soumettre au verdict de l'expérience. Aucun fait n'apparaissant susceptible d'infirmer clairement la théorie, il en vint à mettre en doute la possibilité de donner un réel contenu empirique à l'hypothèse selon laquelle les gens sont rationnels au sens de la théorie de la décision (p. 356). Bien qu'ils se soient soldés par un échec, ces premiers travaux ont laissé des traces durables dans l'œuvre du philosophe américain : le travail en laboratoire n'est pas la seule façon de pratiquer la psychologie, et l'intérêt de Davidson pour la nature de la rationalité ne s'est jamais démenti. Mais s'il renonçait à une carrière de psychologue expérimental, ce n'était pas pour emboîter le pas à ceux qui proposaient de séparer le monde des causes et celui des raisons. Le sens commun est dans le vrai, pour qui croyances et désirs ne deviennent des raisons qu'à condition d'être cause de nos actes, et il n'y a donc pas lieu d'abandonner l'idéal d'une explication causale du comportement. Cette fin de non-recevoir signifiée aux wittgensteiniens était moins novatrice que conservatrice. Davidson s'y présentait comme le champion de l'orthodoxie positiviste et Hempel à la même époque soutenait également, contre les mêmes adversaires, que l'explication par les raisons ne diffère pas, dans ses caractéristiques logiques, de l'explication en général. Les positions des deux auteurs sont pourtant loin de se confondre et le premier, constatant que le schéma d'explication nomologico-déductif développé par le second s'appliquait mal dans le cas des actions, a proposé de réviser à la baisse l'idéal d'explication causale en psychologie. Bref, si Davidson a fait cuvre originale en théorie de l'action, c'est qu'il n'a relié cause et raison que pour dissocier cause et loi.

Les effets de cette décision inaugurale s'étendent bien au-delà de la théorie de l'action. Elle permet de rendre compte des échecs répétés de ceux qui rêvent de constituer les sciences de l'homme sur le modèle des sciences de la nature (p. 307); et c'est sur elle également que s'appuie Davidson pour plaider en faveur du monisme anomal. L'argument fait intervenir trois principes et une thèse :

(1) le principe de l'interaction causale : le mental peut agir sur le physique et réciproquement ; 
(2) le principe du caractère nomologique de la causalité : il n'y a pas de cause sans loi;

(3) le principe de l'anomie du mental : il n'y a pas de loi psychophysique stricte qui permettrait de prédire les événements mentaux et d'en expliquer la nature;

(4) la thèse du monisme anomal est un matérialisme affaibli. Un matérialisme, puisqu'elle admet sans réserve l'identité de la matière et de l'esprit; affaibli, en ce que l'acceptation du dernier principe enveloppe l'abandon d'une idée, souvent tenue pour indissociable de la précédente, selon laquelle le monde de l'esprit est soumis aux lois du monde matériel. Les affirmations (1) et (2) étant supposées bien établies, l'attention se concentre tout entière sur les deux autres, qu'il convient donc de ne pas confondre : l'anomie ne vaut que pour le mental alors que le monisme porte, lui, sur les rapports du mental et du physique. La démarche de Davidson ne leur accorde d'ailleurs pas du tout le même statut. Son but est de montrer que (4) se conclut des trois principes pris comme prémisses, et la construction de la preuve se résumera pour l'essentiel en l'établissement de (3).

L'anomie du mental, loin d'aller de soi comme les deux autres principes, semble en effet incompatible avec leur acceptation. S'il y a interaction causale et s'il n'y a pas de cause sans loi, la logique nous contraint, semble-t-il, à reconnaître que le monde de l'esprit n'échappe pas au règne de la loi. En réalité il s'agit d'un non sequitur, lié à un emploi abusif du deuxième principe, qu'il convient d'« interpréter avec prudence " (p. 288). Une fois dissipée l'équivoque, il restera encore à établir que, dans le cas du mental, on ne peut conclure de la cause à la loi, et l'auteur ne ménage pas ses efforts pour convaincre son lecteur de l'impossibilité de lois psychophysiques strictes. À s'en tenir là, il n'y aurait toutefois guère plus que la répétition, dans un cadre plus vaste, de la dissociation de la cause et de la loi déjà opérée en théorie de l'action. En possession de toutes les prémisses, il reste donc à en déduire la conclusion annoncée. L'argument dira : un événement mental, en tant qu'il entre dans une relation causale (par (1)), tombe sous une loi (par (2)), qui ne peut être que physique (par (3)) et est donc identique à un événement physique (p. 301).

Le monisme anomal a pour tâche de « réconcilier les trois principes » (p. 288) ou, si l'on préfère, de rétablir la situation. En corrigeant l'interprétation erronée du deuxième principe, on risque en effet d'avoir trop prouvé et d'avoir mis en danger le monisme, ce qui n'entre pas du tout dans les intentions de l'auteur. La force de la version affaiblie qu'il en propose vient de ce qu'elle retourne en quelque sorte la valeur argumentative de l'anomie, et utilise en faveur du monisme un fait qui, à première vue, servirait plutôt à le réfuter. Ce matérialisme s'accommode donc de l'irréductibilité du mental. Entre le psychique et le physique, il y a à la fois dépendance causale et indépendance nomologique, et c'est pour caractériser ce lien, plus fort que la dépendance et moins fort que la réduction, qu'on parle de survenance. Faute de pouvoir entrer davantage dans les détails de l'argument, il suffira de signaler que ce sont pour l'essentiel les mêmes considérations qui servent à établir (3) et à conclure de (3) à (4). Le holisme emprunté à Quine y joue un rôle prépondérant. Un même événement peut être décrit en termes physiques ou en termes psychiques. Mais si les deux vocabulaires « ne sont pas faits l'un pour l'autre " (p. 293), c'est que l'univers du mental est seul holiste, et qu'il l'est par essence. On ne peut attribuer à quelqu'un une croyance sans lui en attribuer une foule d'autres, et notre idée 
de rationalité nous interdit de supposer que l'ensemble de ces croyances puisse être totalement incohérent.

L'ouvrage ne se résume bien entendu pas à l'établissement de ces deux thèses et les essais sur l'incontinence, ou sur la forme logique des phrases d'action, ont par exemple donné chacun lieu à de vives discussions. Pris dans son ensemble, il pose deux problèmes majeurs. Dans quelle mesure peut-on tout d'abord faire fonds sur le concept d'action? L'auteur, lorsqu'il tente de déterminer la marque distinctive de l'action, n'évoque les doutes d'Austin, qui estimait la question mal posée, que pour les écarter et adopter l'hypothèse opposée. Rétrospectivement, il admet toutefois que dans l'essai $n^{\circ} 3$ «l'exigence essentielle, celle d'une analyse de l'agir (et par conséquent [...] de l'action) n'est pas satisfaite" (p. 7). La difficulté se cristallise autour de ce qu'on a appelé l'effet accordéon. En marchant, j'ai tiré par mégarde sur un fil électrique, fait sauter le transformateur du quartier, provoqué un accident mortel au carrefour et bouleversé pour une soirée la vie de centaines de foyers. Une fois remis de l'étonnement où l'on ne peut manquer d'être plongé à découvrir que l'art de faire plusieurs choses à ja fois est à la portée du premier venu, reste à s'entendre sur la nature exacte du phénomène. La possibilité d'étendre ou de contracter à volonté l'action rend d'incontestables services. En nous amenant à nous demander s'il s'agit d'autant d'actions différentes, ou d'une seule et même action décrite en des termes différents, elle offre un argument supplémentaire en faveur de l'approche linguistique chère aux philosophes analytiques: "Pour clarifier les choses, nous devons parler non pas d'actions mais plutôt de phrases ou de descriptions d'action " (p. 71). Ainsi l'établissement de (3) repose sur l'indifférence des événements à leur mode de description : un même événement peut être décrit tout à tour en termes physiques et psychiques. Mais la situation a aussi des effets dévastateurs. Où commence et où finit l'action? Si l'on accepte qu'un agent cause ce que causent ses actes, on court le risque de ne plus pouvoir distinguer ceux-ci de leurs conséquences, et de diluer l'action dans le cours des événements. Pour briser net la difficulté, l'auteur isole une classe d'actions dites primitives qui seront les seules à être d'authentiques actions. "Nous ne faisons jamais autre chose que mouvoir notre corps : c'est la nature qui se charge de faire tout le reste » (p. 89). L'inclusion des conséquences n'a lieu que dans les descriptions, et non dans l'action, qui reste invariablement la même, indépendante qu'elle est et de ses conséquences et de ses descriptions. Davidson dissimule toutefois mal son embarras et n'échappe pas, semble$\mathrm{t}-\mathrm{il}$, au reproche qu'il adresse à ce propos à Austin puisque, en même temps qu'il reconnaît la prégnance de l'effet accordéon, au point d'être prêt à y voir la marque distinctive de l'agir, il n'a rien de plus pressé que de dénoncer les confusions fondamentales qui lui sont associées. Ces incertitudes rejaillissent sur le statut de la théorie tout entière. Certes elle peut se réclamer de l'autorité d'Aristote; mais il y a loin de l'action rationnelle à la raison pratique, et l'agent rationnel ressemble peu au sage antique. Si l'anomie du mental n'implique pas son autonomie (p. 277), il faudrait du moins s'assurer qu'elle ne mène pas à cette anarchie mentale dont on sait par ailleurs ce qu'il faut penser. La philosophie de l'action hésite, sans jamais se prononcer clairement, entre la morale et la psychologie. Bien que l'attribution de la rationalité soit normative, le point de vue adopté reste dans son ensemble descriptif et l'on se demande s'il ne s'agirait pas d'une morale qui aurait honte de se présenter pour ce qu'elle est et préférerait se retrancher derrière des considérations psychologiques. 
Le second problème est d'un autre ordre. Davidson manifeste une préférence marquée pour les théories modestes, les positions affaiblies. Mais que faut-il penser d'une théorie du langage qui, tout en reconnaissant la nature intentionnelle des langues, choisit de s'en tenir au point de vue de l'extension, ou d'une théorie de l'esprit qui admet d'emblée n'avoir «pas réussi à saisir notre concept intuitif de mental» (p. 283) ? Certes la nécessité est mère de l'invention, et Davidson exploite avec une rare ingéniosité les maigres ressources qu'il s'autorise à utiliser. Certains mettront également à son crédit l'honnêteté et la modestie intellectuelles qui font qu'il est le premier à signaler les limites ou les insuffisances de ses théories. D'autres, en revanche, s'étonneront que tant d'espoirs aient été mis dans des propositions qui semblaient se condamner par avance à l'échec et demanderont de ne pas prendre pour acquis que la politique des demi-mesures soit la mieux à même de nous rapprocher de la vérité. Heureusement, il n'est pas nécessaire d'être d'accord avec toutes les thèses défendues par l'auteur, ni d'être victime d'américanomanie, pour reconnaître qu'il s'agit d'une contribution de premier plan. Ce n'est pas d'aujourd'hui que la philosophie s'interroge sur la causalité ou sur les rapports de l'âme et du corps, et l'on aimerait lire plus souvent des ouvrages de cette qualité. Pascal Engel a joint une présentation, qui permet au lecteur de s'orienter dans les essais qui la suivent et donne un aperçu des nombreux débats qu'ils ont suscités. La traduction aurait gagné à être faite avec plus de soin et il faut trop souvent pour comprendre se rapporter à l'original. En dépit de ces négligences, auxquelles il n'aurait pas été difficile de remédier, le public francophone saura gré au traducteur de lui avoir permis de juger sur pièce de l'œuvre d'un des plus en vue parmi les philosophes du Nouveau Monde.

Michel BourdeAu

\section{CULTURES ET HISTOIRE}

La Rome des premiers siècles. Légende et histoire. Actes de la table ronde en l'honneur de Massimo Pallotrino, Paris, 3-4 mai 1990. Florence, Leo S. Olschki, 1992. $19 \times 27,5$, VII-270 p., index, pl. («Istituto nazionale di studi etruschi e italici. Studi etruschi », 24).

C'est un bel hommage que la Section française de l'Institut national d'études étrusques et italiques a rendu à son président Massimo Pallottino en organisant, en liaison avec le Laboratoire d'archéologie de l'École normale supérieure et l'Équipe 